\title{
The receptor tyrosine kinase EphA2 promotes mammary adenocarcinoma tumorigenesis and metastatic progression in mice by amplifying ErbB2 signaling
}

Dana M. Brantley-Sieders, ${ }^{1}$ Guanglei Zhuang, ${ }^{2}$ Donna Hicks, ${ }^{1}$ Wei Bin Fang, ${ }^{2}$ Yoonha Hwang, ${ }^{1}$ Justin M.M. Cates, ${ }^{3}$ Karen Coffman, ${ }^{4}$ Dowdy Jackson, ${ }^{4}$ Elizabeth Bruckheimer, ${ }^{4}$ Rebecca S. Muraoka-Cook, 5 and Jin Chen $1,2,6,7$

\begin{abstract}
1Department of Medicine, Division of Rheumatology and Immunology, 2Department of Cancer Biology, and 3Department of Pathology, Vanderbilt University School of Medicine, Nashville, Tennessee, USA. ${ }^{4}$ MedImmune Inc., Gaithersburg, Maryland, USA.

${ }^{5}$ Lineberger Comprehensive Cancer Center, Department of Genetics and Radiation Oncology, University of North Carolina, Chapel Hill, North Carolina, USA. ${ }^{6}$ Vanderbilt-Ingram Cancer Center, and 7Department of Cell and Developmental Biology, Vanderbilt University School of Medicine, Nashville, Tennessee, USA.
\end{abstract}

\begin{abstract}
Overexpression of the receptor tyrosine kinase $\mathrm{EPH}$ receptor $\mathrm{A2}$ (EphA2) is commonly observed in aggressive breast cancer and correlates with a poor prognosis. However, while EphA2 has been reported to enhance tumorigenesis, proliferation, and MAPK activation in several model systems, other studies suggest that EphA2 activation diminishes these processes and inhibits the activity of MAPK upon ligand stimulation. In this study, we eliminated EphA2 expression in 2 transgenic mouse models of mammary carcinoma. EphA2 deficiency impaired tumor initiation and metastatic progression in mice overexpressing ErbB2 (also known as Neu) in the mammary epithelium (MMTV-Neu mice), but not in mice overexpressing the polyomavirus middle T antigen in mammary epithelium (MMTV-PyV-mT mice). Histologic and ex vivo analyses of MMTV-Neu mouse mammary epithelium indicated that EphA2 enhanced tumor proliferation and motility. Biochemical analyses revealed that EphA2 formed a complex with ErbB2 in human and murine breast carcinoma cells, resulting in enhanced activation of Ras-MAPK signaling and RhoA GTPase. Additionally, MMTV-Neu, but not MMTV-PyV-mT, tumors were sensitive to therapeutic inhibition of EphA2. These data suggest that EphA2 cooperates with ErbB2 to promote tumor progression in mice and may provide a novel therapeutic target for ErbB2-dependent tumors in humans. Moreover, EphA2 function in tumor progression appeared to depend on oncogene context, an important consideration for the application of therapies targeting EphA2.
\end{abstract}

\section{Introduction}

Malignant progression of solid tumors is a complex process that involves the activation of oncogenic signaling and downregulation of tumor suppressor pathways. In addition, modulation of the tumor microenvironment, for example through neovascularization, enhances tumor cell growth and survival, promoting invasion and metastatic spread (reviewed in refs. 1-3). Oncogenic conversion, amplification, or overexpression of protooncogenes, such as those encoding cell surface receptor tyrosine kinases (RTKs) like the EGF receptor family member ErbB2, are frequently observed in human cancers and contribute to malignancy. Other pathways, such as p53 transcription factor/genome surveillance factor, negatively regulate growth, and loss of these pathway components also contributes to tumorigenesis (reviewed in refs. 3, 4). Recent evidence suggests that Eph RTKs play multiple roles in neoplastic progression, including regulation of processes intrinsic to tumor cells, and in the tumor microenvironment, such as tumor neovascularization (reviewed in refs. 5-8).

Nonstandard abbreviations used: EphA2, EPH receptor A2; HER2, human ErbB2; MMTV, mouse mammary tumor virus; PCNA, proliferating cell nuclear antigen; PMEC, primary mammary epithelial cell; PMTC, primary mammary tumor cell; $\mathrm{PyV}-\mathrm{mT}$, polyoma virus middle T; RTK, receptor tyrosine kinase.

Conflict of interest: The authors have declared that no conflict of interest exists. Citation for this article: J. Clin. Invest. 118:64-78 (2008). doi:10.1172/JCI33154.
The Eph RTK family is the largest family of RTKs identified in the genome, with at least 15 receptors and 9 ligands identified in vertebrates (reviewed in refs. 5,9 ). The family is subdivided into class A and class B based on homology and binding affinity for 2 distinct types of membrane-anchored ephrin ligands. Class B receptors generally bind to class $B$ ephrins that are attached to the cell membrane by a transmembrane-spanning domain, while A class receptors normally interact with glycosyl-phosphatidylinositol-linked class A ephrins, although interclass binding does occur among certain family members (reviewed in refs. 5, 9). These molecules function during embryogenesis to regulate angiogenic remodeling processes, axon guidance, and tissue boundary formation (reviewed in refs. 10,11). More recently, members of this RTK family, including EPH receptor A2 (EphA2), have been linked to tumor progression and neovascularization (reviewed in ref. 6).

Increasing evidence suggests that EphA2 expression may be causally related to neoplasia. EphA2 RTK overexpression has been observed in several models of cancer, including primary and transplanted rodent tumors, human tumor xenografts, and primary human tumor biopsies (reviewed in refs. 5-7). Experimentally induced overexpression of EphA2 resulted in malignant transformation of nontransformed MCF10A breast cells and enhanced malignancy of pancreatic carcinoma cells $(12,13)$. Conversely, siRNA-mediated inhibition of EphA2 expression impaired malignant progression of pancreatic, ovarian, and mesothelioma 


\section{Table 1}

Incidence of hyperplasia, tumorigenesis, and lung metastasis frequency in mice 8 months and 1 year after birth

\begin{tabular}{lcccc} 
Genotype & Hyperplasia & \multicolumn{2}{c}{ Tumors } & Lung lesions \\
& $\mathbf{8} \mathbf{~ m o}$ & $\mathbf{8} \mathbf{~ m o}$ & $\mathbf{1} \mathbf{~ y r}$ & $\mathbf{1} \mathbf{~ y r}$ \\
EphA2+/+ MMTV-Neu & $71 \%$ & $57 \%$ & $72 \%$ & $63 \%$ \\
EphA2+/- MMTV-Neu & $64 \%$ & $43 \%$ & $76 \%$ & $69 \%$ \\
EphA2-/- MMTV-Neu & $23 \%$ & $23 \%{ }^{\mathrm{A}}$ & $40 \%{ }^{\mathrm{A}}$ & $44 \%$
\end{tabular}

$n=13-14$ per genotype scored 8 months after birth; 10-13 per genotype scored 1 year after birth. ${ }^{A} P<0.05$ versus EphA2 ${ }^{+/+}$MMTV-Neu; $\chi^{2}$ test.

tumor cell lines, and overexpression of dominant-negative EphA2 constructs suppressed growth and metastasis of 4T1 metastatic mouse mammary adenocarcinoma cells in vivo (13-16). EphA-Fc receptor proteins that disrupt endogenous receptor activation significantly inhibited growth and neovascularization of tumors in vivo (17-19). Coupled with the observation that EphA2 signaling induces phosphorylation and activation of the pro-proliferative p42/44 MAPK family member Erk in tumor cell lines (20,21), these data suggest that EphA2 functions as an oncogene.

Other evidence, however, suggests that EphA2 may function as a tumor suppressor. EphA2-/- gene-trap mice displayed increased susceptibility to chemical carcinogen-induced skin cancer compared with control littermates, along with increased tumor cell proliferation and phosphorylation of Erk (22). Stimulation of EphA receptors with soluble ephrin-A1-Fc ligand reduced Erk phosphorylation in tumor cell lines, fibroblasts, and primary aortic endothelial cells and suppressed growth of primary keratinocytes and prostate carcinoma cells (22-24). Macrae et al. also reported that treatment of human breast cancer cell lines with ephrin-A1-Fc, which stimulated EphA2 phosphorylation, attenuated EGF-mediated phosphorylation of Erk and inhibited transformation of NIH3T3 cells expressing v-erbB2 (24). In addition, EphA2 was reported to be a transcriptional target of the tumor suppressor p53 (25-28). Overexpression of EphA2 in lung and breast cancer cell lines negatively regulated proliferation and induced apoptosis $(25,28)$. These data suggest that EphA2 functions as a tumor suppressor.

Given the controversy surrounding the role of EphA2 in tumorigenesis, we investigated the consequences of EphA2 deficiency in transgenic mouse models of endogenous mammary tumor formation. We chose the MMTV-Neu and MMTV-PyV-mT transgenic models, as they each recapitulate the numerous stages of human breast tumor formation and progression. In these models, the mouse mammary tumor virus (MMTV) long-terminal repeat drives expression of Neu, the rat homolog of ErbB2, or polyoma virus middle $\mathrm{T}(\mathrm{PyV}-\mathrm{mT})$ antigen specifically in mammary gland epithelium. These models recapitulate multistage tumor progression in vivo in a similar fashion to that observed in human breast cancer, making them excellent models for analysis of endogenous tumor progression $(29,30)$. Herein, we demonstrate that EphA2 enhanced tumor formation and proliferation in the context of Neu, both in vivo and in ex vivo molecular analyses of purified tumor cells. Hostderived EphA2 was required for maximal tumor vascularization in the MMTV-Neu model. Within MMTV-Neu tumor cells, maximal levels of Neu/ErbB2 signaling required EphA2, which promoted both tumor initiation and metastatic progression of MMTVNeu-derived mammary tumors. Therapeutic inhibition of EphA2 impaired growth of MMTV-Neu tumors. By contrast, EphA2 deficiency or inhibition did not affect tumor formation or progression in the MMTV-PyV-mT model of breast cancer (29). These results demonstrate that the role of EphA2 in tumor progression is dependent upon the oncogene/tumor suppressor context within which it functions. Such considerations are likely to be important regarding the application of therapies targeting EphA2.

\section{Results}

EphA2 deficiency suppresses mammary epithelial byperplasia, tumorigenesis, and metastasis in MMTV-Neu mice. MMTV-Neu-positive female mice that were $\mathrm{EphA} 2^{+/+}$, EphA2 $2^{+/}$, or EphA2 $2^{-/-}$were generated and monitored for tumor formation. Mammary gland tissue and/or tumors were collected from 2 cohorts of animals 8 months and 1 year after birth. Relative to EphA2 $2^{+/}$and EphA2 $2^{+/}$controls, EphA2 $2^{-/}$ MMTV-Neu females exhibited a significant decrease in epithelial hyperplasias and tumors of the mammary gland with a 2-to 3-fold reduction in frequency (Table 1 ). Whole-mount and histologic analysis revealed a reduction in mammary epithelial hyperplasia and epithelial cell content for EphA2-/- MMTV-Neu glands relative to controls (Figure 1B). In fact, the mammary epithelium failed to penetrate the mammary fat pad in $30 \%$ of EphA2 -/- MMTV-Neu mammary glands (Supplemental Figure 1A; supplemental material available online with this article; doi:10.1172/JCI33154DS1).

To examine premalignant changes within the epithelium of EphA2 -/- versus EphA2 ${ }^{+/+}$MMTV-Neu mammary glands, we assessed proliferation and apoptosis in tissue sections by staining for proliferating cell nuclear antigen (PCNA) and by TUNEL assay, respectively. We observed a 5.5-fold reduction in epithelial cell proliferation in the EphA2 $2^{-/-}$versus the EphA2 $2^{+/+}$MMTV-Neu mammary epithelium, while levels of apoptosis were unaffected (Figure 1C). To determine whether proliferation defects were due to EphA2 deficiency in mammary epithelium versus surrounding host tissue, we analyzed proliferation and apoptosis in purified primary mammary epithelial cells (PMECs) isolated from EphA2 ${ }^{+/+}$or EphA2 $2^{-/-}$animals. Proliferation, as measured by incorporation of BrdU, was reduced nearly 3-fold in serum-stimulated EphA2 $2^{-/}$cells relative to EphA2 ${ }^{+/+}$controls (Figure 1D), suggesting that EphA2-mediated effects on proliferation are, at least in part, intrinsic to the epithelial cell. Interestingly, unlike mammary epithelium in situ, we observed a modest yet significant increase in apoptosis for EphA2 $2^{-/-}$versus EphA2 $2^{+/}$PMECs (Figure 1D). Together, these data indicate that loss of EphA2 inhibits ErbB2initiated mammary epithelial cell hyperplasia.

Among the EphA2 $2^{--}$animals that actually developed tumors, no significant change in time of tumor onset was observed. However, we detected a nearly 3 -fold decrease in tumor volume in EphA2-/relative to $\mathrm{EphA} 2^{+/+}$mice (data not shown). In addition, EphA2 ${ }^{+/+}$ and EphA2 $2^{+-}$controls displayed a higher overall tumor burden relative to EphA2-/- mice, as control animals developed 2 or more tumors 1 year after birth while EphA2 ${ }^{-/-}$animals developed single tumors. While EphA2 protein expression was detected in mammary epithelial cells, associated blood vessels, and total mammary gland lysates in EphA2 $2^{+/}$and EphA2 $2^{+-}$MMTV-Neu females, EphA2 protein expression was not detected in tissue or mammary gland lysates harvested from EphA2-/- MMTV-Neu mice (Supplemental Figure 1B). Expression levels and localization of ErbB2 in MMTVNeu tumors were not affected by EphA2 deficiency (Supplemental Figure 1C), although EphA2 expression appeared to be elevated in mammary gland lysates isolated from 8-month-old MMTV-Neu 


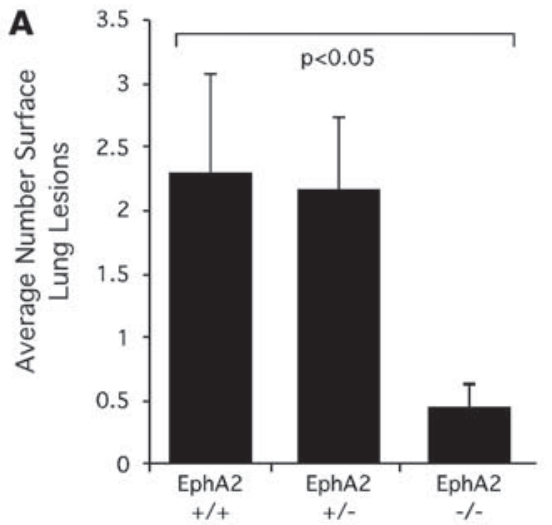

B MMTV-Neu/EphA2 $2^{+/+}$MMTV-Neu/EphA2+/- MMTV-Neu/EphA2\%

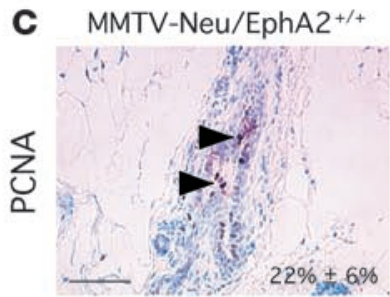

MMTV-Neu/EphA2
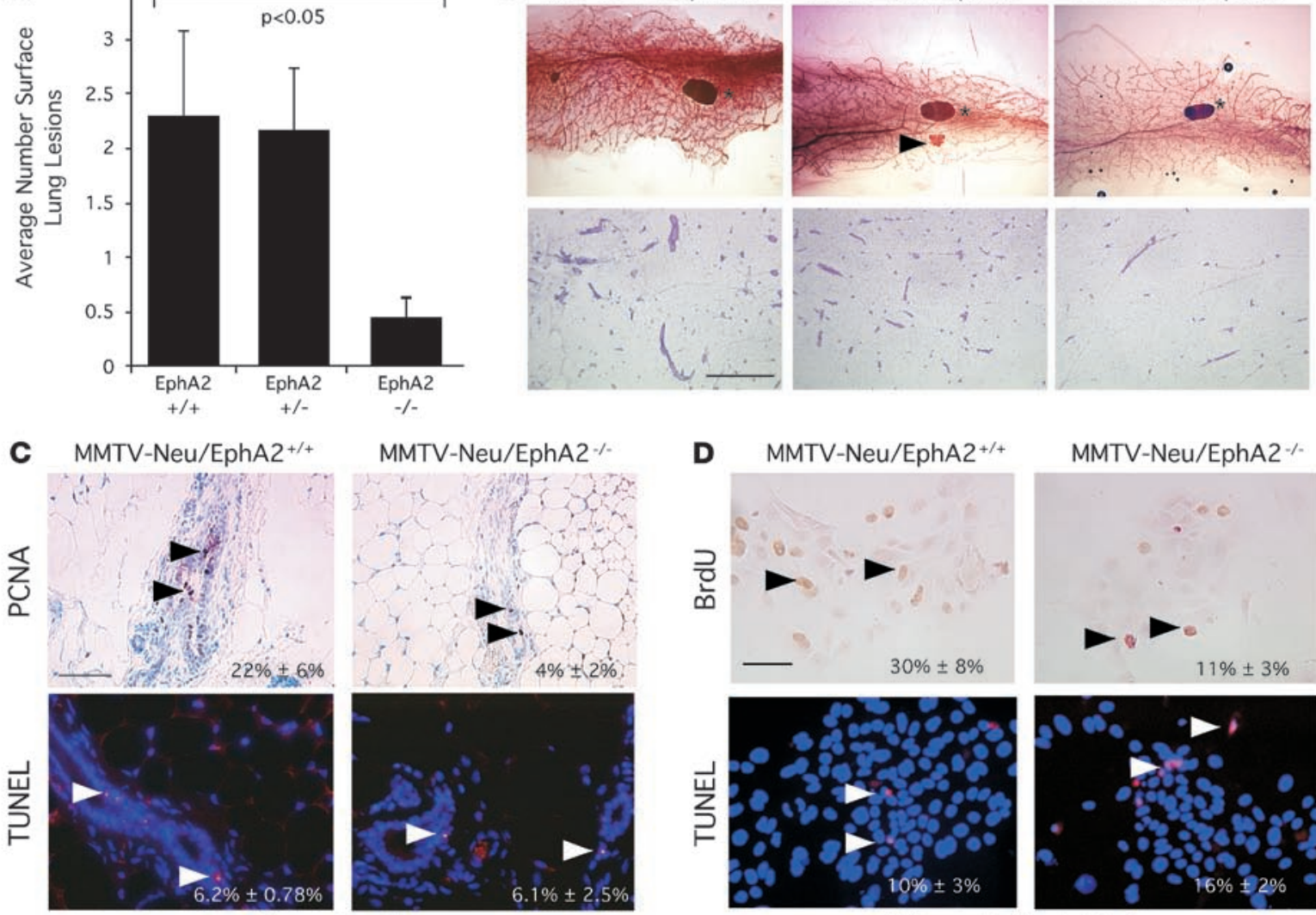

MMTV-Neu/EphA2

Tissue Sections
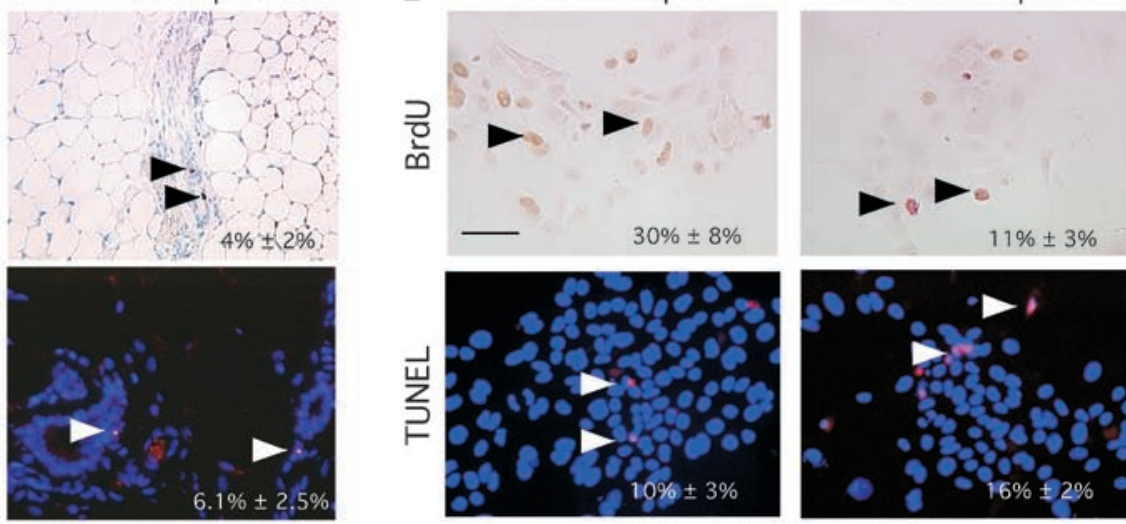

Primary Epithelial Cells

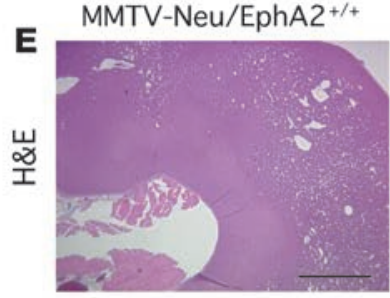

MMTV-Neu/EphA2+/-

MMTV-Neu/EphA2 \%
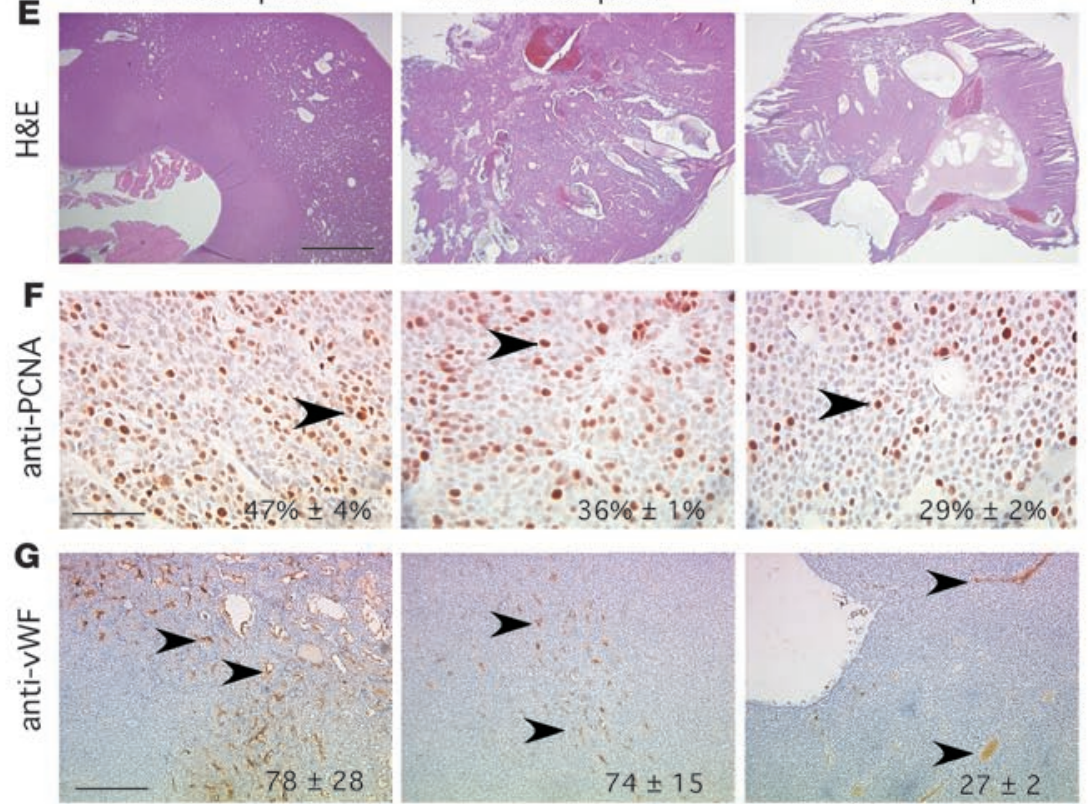


\section{Figure 1}

EphA2 deficiency reduces mammary tumorigenesis, metastasis, proliferation, and vascularity in MMTV-Neu mice. (A) Number of surface lung lesions was significantly reduced in EphA2 ${ }^{-/-}$MMTV-Neu mice $(P<0.05$; single-factor ANOVA). Data are mean \pm SEM. (B) Top: Whole-mount mammary gland preparations $(8 \mathrm{mo})$ revealed diminished hyperplasia in $\mathrm{EphA}^{-/-}$glands relative to controls. Shown are an $\mathrm{EphA}^{+/+}$gland with pervasive epithelial hyperplasia (left) and an EphA2 $2^{+-}$gland with a small tumor (arrowhead; middle). Asterisks indicate inguinal lymph node. Bottom: H\&E-stained mammary gland sections $(8 \mathrm{mo})$ reveal reduced epithelial cell content in EphA2 $2^{-/-}$ MMTV-Neu tissue samples relative to controls. Scale bar: $250 \mu \mathrm{m}$. (C) Top: Mammary epithelial proliferation (PCNA+ nuclei; arrowheads), was significantly reduced ( $P<0.05$; 2-tailed, paired Student's $t$ test). Scale bar: $50 \mu \mathrm{m}$. Bottom: Mammary epithelial apoptosis (TUNEL+ nuclei; arrowheads) was not affected. (D) Top: Proliferation of primary mammary epithelial cells from EphA2-/- animals (BrdU incorporation; arrowheads) was reduced relative to $\mathrm{EphA} 2^{+/+}$cells $(P<0.05$; 2-tailed, paired Student's $t$ test). Bottom: Apoptosis (TUNEL+ nuclei; arrowheads) was significantly increased in EphA2 ${ }^{--}$primary mammary epithelial cells relative to controls $(P<0.05$; 2-tailed, paired Student's $t$ test). Scale bar: $20 \mu \mathrm{m}$. (E) H\&E-stained tumor sections (1 yr) demonstrate increased cystic degeneration and lumen formation in EphA2 ${ }^{-1}$ tumors. Scale bar: $250 \mu \mathrm{m}$. (F) Decreased tumor cell proliferation $\left(\mathrm{PCNA}^{+}\right.$nuclei; arrowheads) was observed for EphA2 $2^{-1}$ MMTV-Neu tumors compared with controls ( $P<0.05$; single-factor ANOVA). Scale bar: $50 \mu \mathrm{m}$. (G) Microvascular density (CD31+ vessels; arrowheads) was significantly reduced in EphA2 $2^{-/-}$MMTV-Neu tumors relative to controls $(P<0.05$; single-factor ANOVA). Scale bar: $100 \mu \mathrm{m}$.

mice relative to FVB controls (Supplemental Figure 1C). At 1 year of age, lungs harvested from EphA2-/- MMTV-Neu mice displayed a nearly 5 -fold reduction in the number of surface metastases compared with EphA2 ${ }^{+/}$or EphA2 ${ }^{+/}$controls (Figure 1A). Moreover, the overall frequency of metastasis was decreased in EphA2 $2^{--}$animals relative to EphA2 ${ }^{+/+}$and EphA2 $2^{+/-}$controls (Table 1 ).

Histologic examination of tumors collected from each genotype 8 months after birth disclosed mainly well-circumscribed proliferations of invasive carcinoma with broad pushing, rather than infiltrating, borders. More infiltrative-appearing carcinomas were seen in animals 1 year after birth. Tumors isolated from EphA2-/MMTV-Neu mice showed more areas of cystic degeneration and occasional lumen formation, suggestive of a more differentiated phenotype relative to the dense, solid sheet-like growth patterns seen in EphA2 $2^{+/+}$MMTV-Neu tumors (Figure 1E). PCNA staining of tumor tissue revealed a nearly 2 -fold decrease in proliferation in EphA2-/- relative to EphA2 ${ }^{+/+}$MMTV-Neu tumors (Figure $1 \mathrm{~F}$ ). The tumor microvasculature was evaluated by immunohistochemical staining against vWF, which demonstrated that loss of EphA2 expression was associated with a significant 2.9-fold reduction in microvascular density (Figure 1G). Levels of apoptosis were unaltered in EphA2-/- MMTV-Neu tumors compared with controls (data not shown). These data suggest that EphA2 is required for both mammary tumor initiation and progression.

EPhA2 is required in the host microenvironment for vascular recruitment in MMTV-Neu tumors. While the data presented herein suggest that EphA2 deficiency restrains epithelial proliferation in MMTV-Neu mammary glands, previously reported data suggest that EphA2 may be required for tumor vascularization (reviewed in ref. 5). Indeed, decreased tumor vascularization was observed in EphA2-MMTV-Neu tumors (Figure 1G). To determine whether the defects in tumor microvascular density result from EphA2 deficiency in host tissue versus tumor cells, we orthotopically transplanted EphA2 $2^{+/}$MMTV-Neu tumor cells (31) into the cleared fat pads of syngeneic EphA2 $2^{+/+}$or EphA2-/- FVB host animals. EphA2 $2^{+/+}$tumor cells transplanted into EphA2/- hosts produced significantly smaller tumors than those transplanted into EphA2 ${ }^{+/}$hosts (Supplemental Figure 2A). We also observed a 7 -fold decrease in microvascular density of tumors isolated from EphA2 $2^{-/-}$versus EphA2 $2^{+/+}$ recipients (Supplemental Figure 2B). Consistent with these data, microvascular endothelial cells isolated from EphA2-/- animals displayed a markedly decreased migratory response to MMTV-Neu tumor cells in coculture assays compared with the robust migratory response exhibited by endothelial cells isolated from EphA2 $2^{+/+}$ mice (Supplemental Figure 2C). Together, these data suggest that EphA2 signaling promotes tumorigenesis and progression through distinct processes both in the tumor microenvironment, including vascular endothelium, and within tumor cells.

Loss of EphA2 expression impairs tumor formation and invasiveness in MMTV-Neu tumor cells. In addition to analysis of EphA2 function in tumor initiation and progression within endogenous MMTVNeu tumors in which EphA2 deficiency precedes tumorigenesis, we examined the effects of diminishing EphA2 expression in established tumor cells. Using an RNAi knockdown strategy in an established cell line derived from an MMTV-Neu tumor (31), stable expression of 2 independent siRNA sequences significantly reduced EphA2 expression in MMTV-Neu cells relative to parental cells and cells expressing control siRNA (Figure 2A). Pooled populations of cells in which EphA2 expression was diminished displayed slower growth rates than parental or control siRNA-expressing cells (data not shown). Consistent with the diminished growth rates, inhibition of EphA2 expression by siRNA correlated with diminished levels of p-Erk, a known regulator of proliferation in the MMTV-Neu model (reviewed in ref. 32), in EphA2 siRNA clones (Figure 2A). Parental MMTV-Neu cells and cells transduced with the control siRNA formed large, multiacinar structures and failed to form lumens in 3-dimensional Matrigel culture, consistent with previous descriptions of the effects of ErbB2 activity on 3-dimensional cultures of human MCF10A cells (33). In contrast, diminished EphA2 expression impaired the ErbB2/Neu-driven multiacinar phenotype of the MMTV-Neu cells in 3-dimensional culture. Instead, these cells primarily formed small, organized acini composed of epithelial cells surrounding a single central lumen (Figure 2, B and C). Furthermore, the size of individual 3-dimensional colonies formed by control cells was 3- to 4-fold greater than cells with decreased EphA2 expression (Figure 2B). While MMTV-Neu parental cells or cells expressing control siRNAs formed tumors when orthotopically transplanted in the cleared fat pads of FVB recipient female mice, MMTV-Neu cells with diminished EphA2 expression failed to establish tumors or formed very small, nonpalpable tumors in a small percentage of animals (Figure 2D). These data suggest that EphA2 activity is required for tumor cell-intrinsic growth and invasiveness in the context of the ErbB2/Neu oncogene.

Elevated EphA2 expression augments growth and invasiveness of MCF10A cells overexpressing human ErbB2. To determine whether EphA2 enhances ErbB2-mediated growth and invasiveness in human cells, we overexpressed EphA2 in both nontransformed MCF10A human mammary epithelial cells and in MCF10A cells that stably express the human homolog of ErbB2 (HER2; ref. 34) by adenoviral transduction. Consistent with previous studies (12), overexpression of EphA2 enhanced growth, as we observed increased colony size in 3-dimensional Matrigel culture (Figure 3A). 
A

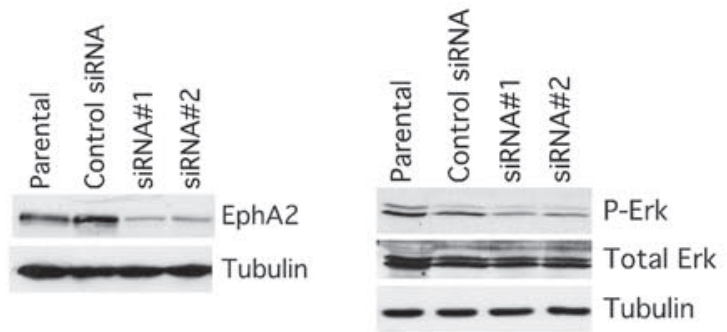

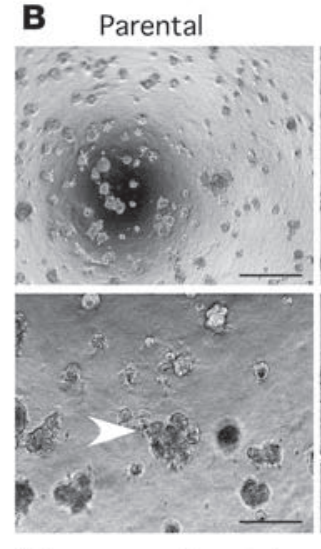

c

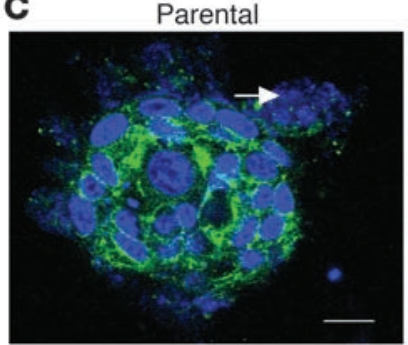

Control siRNA

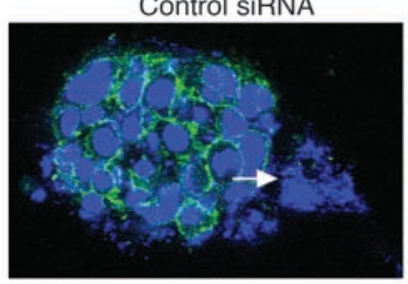

Control siRNA

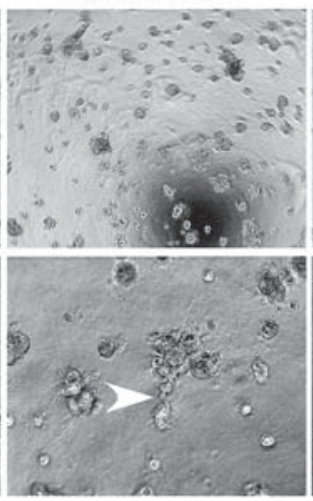

SiRNA\#1

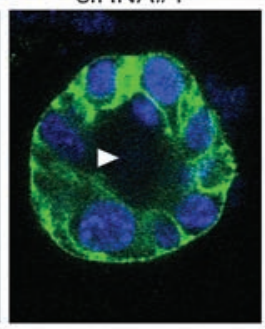

SiRNA\#2

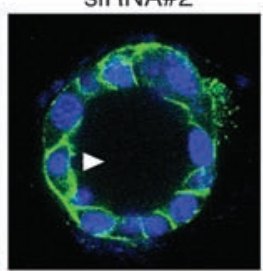

EphA2 siRNA\#1
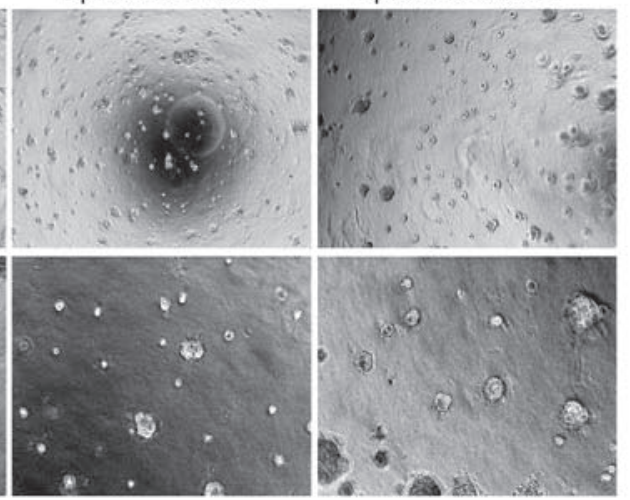

D

EphA2 siRNA\#2

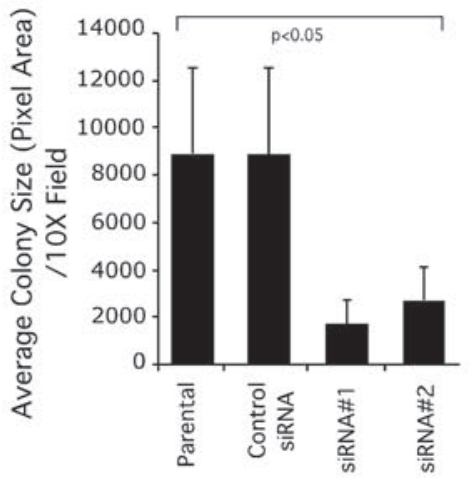

\section{Figure 2}

Loss of EphA2 expression impairs tumor formation and invasiveness in MMTV-Neu tumor cells. (A) EphA2 expression was significantly diminished in MMTV-Neu tumor cells transduced with retroviruses expressing EphA2 siRNA sequences versus control siRNAs. Erk phosphorylation was reduced upon EphA2 knockdown. (B) Parental and control siRNA tumor cells formed large, irregularly shaped clusters with invasive protrusions (arrowheads) when cultured on Matrigel, whereas EphA2 siRNA-expressing cells formed smaller clusters with a rounded morphology and few protrusions, indicative of reduced invasiveness. Scale bar: $200 \mu \mathrm{m}$ (top), $50 \mu \mathrm{m}$ (bottom). We observed a significant decrease in colony size, as determined by calculating the average pixel area occupied by individual colonies, for cells expressing EphA2 siRNA relative to controls $(P<0.05$; single-factor ANOVA). (C) Cultures stained with TO-PRO-3 iodide nuclear stain (blue) and anti-E-cadherin (green) were imaged by confocal microscopy. Control tumor cells formed multiacinar structures with invasive protrusions (arrowheads), whereas tumor cells expressing EphA2 siRNA sequences formed round, uniform acinar structures composed of a single layer of epithelial cells surrounding a central lumen (arrows). Scale bar: $20 \mu \mathrm{m}$. (D) Upon orthotopic transplantation into cleared fat pads of FVB recipient female mice, tumor cells expressing control siRNA sequences produced tumors of comparable volume to those generated by transplantation of parental cells at 5 weeks. Tumor cells expressing EphA2 siRNA sequences, however, either failed to form tumors or formed very small, nonpalpable tumors in a small fraction of animals $(P<0.05$; single-factor ANOVA $)$. Data are mean \pm SEM.

Relative to parental MCF10A, HER2-overexpressing (MCF10A. HER2) cells formed larger, multiacinar structures that failed to form lumens in 3-dimensional Matrigel culture (Figure 3A), consistent with previous reports $(33,34)$. Overexpression of EphA2 by adenoviral transduction in MCF10A.HER2 cells led to a 2-fold increase in the size of individual colonies relative to untransduced controls or cells transduced with adenovirus expressing $\beta$-gal (Figure $3 \mathrm{~A}$ ). In addition, there was an increase in lumen filling and 
A
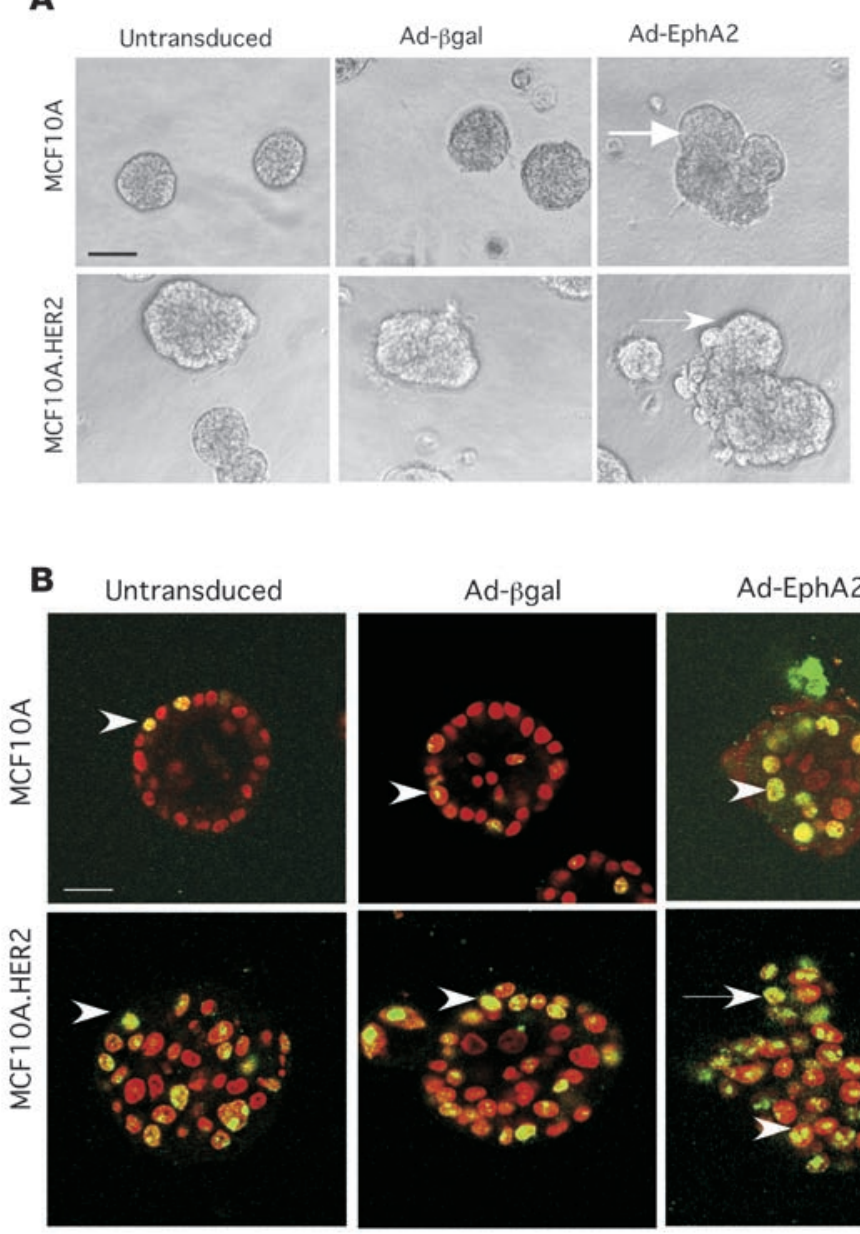

C

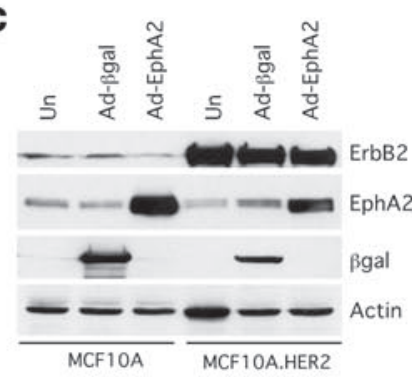

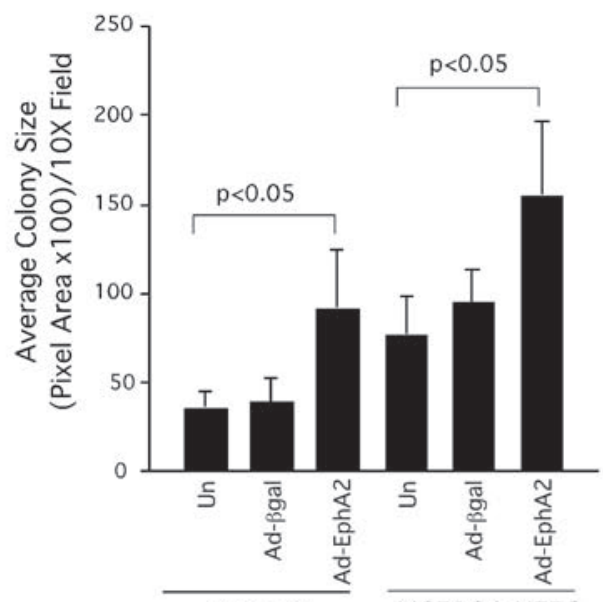

MCF10A Ad-EphA2
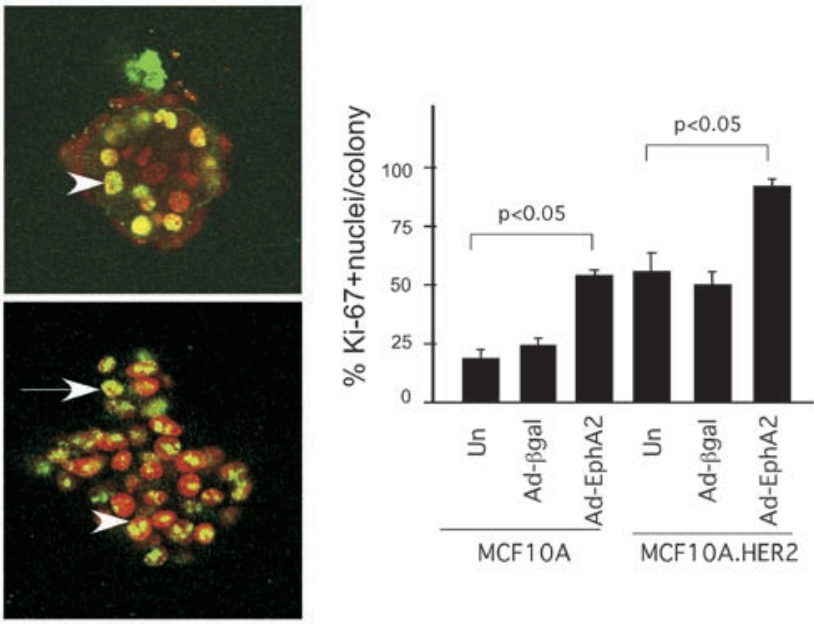

\section{Figure 3}

Elevated EphA2 expression in MCF10A.HER2 cells enhances cell proliferation and invasiveness in vitro. (A) Parental MCF10A human breast cells and MCF10A.HER2 cells were transduced with adenoviruses (Ad) expressing EphA2 or control $\beta$-gal and plated on growth factor-reduced Matrigel to generate 3-dimensional spheroid cultures. After 10 days in culture, parental MCF10A cells and cells expressing Ad- $\beta$-gal formed small, round acinar structures, while MCF10A.HER2 cells formed larger colonies with irregular, invasive protrusions (arrows). Expression of Ad-EphA2 in MCF10A cells resulted in larger, irregular colonies, an effect that was amplified in MCF10A.HER2 cells $(P<0.05$; single-factor ANOVA). Scale bar: $25 \mu \mathrm{m}$. (B) Cultures were stained with TO-PRO-3 iodide nuclear stain (red) and anti-Ki67 (green) and imaged by confocal microscopy. Confocal analysis revealed that parental and Ad- $\beta$-gal-transduced MCF10A formed uniform acinar structures composed of a single layer of epithelial cells surrounding a central lumen, while MCF10A.HER2 cells formed multiacinar structures with invasive protrusions (arrows) and a poorly defined lumen containing several cells. MCF10A cells transduced with Ad-EphA2 also formed multiacinar structures with a poorly defined lumen. Invasion and lumen filling were enhanced in MCF10A.HER2 cells overexpressing EphA2. Scale bar: $20 \mu \mathrm{m}$. EphA2 overexpression significantly enhanced proliferation (Ki67+ nuclei, arrows) within acinar structures formed by MCF10A and MCF10A.HER2 cells $(P<0.05$; single-factor ANOVA). (C) Expression of adenoviral gene products and overexpression of ErbB2/HER2 in MCF10A.HER2 cells was confirmed by immunoblot, and uniform loading was verified by immunoblot for actin. Expression of p-Erk, total Erk, p-EphA2, and total EphA2 was also assessed by immunoblot. 
A

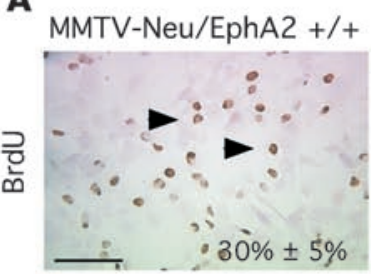

MMTV-Neu/EphA2 -/-

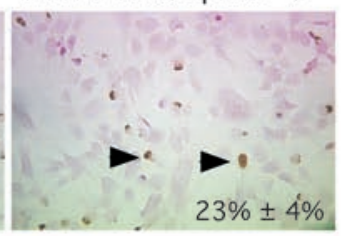

MMTV-Neu EphA2 -/- MMTV-Neu EphA2 -/Ad-Bgal

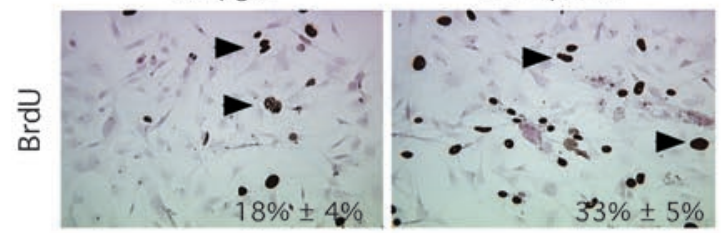

C

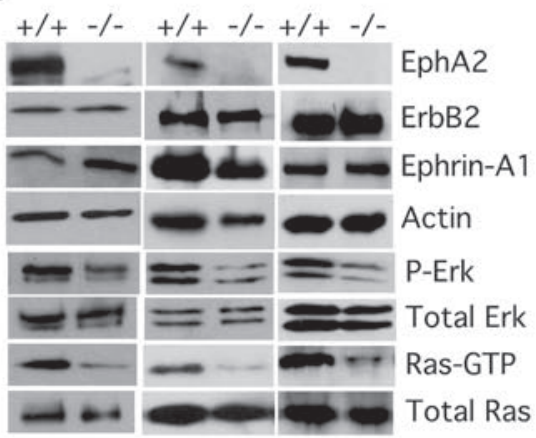

Whole Tumor Extracts

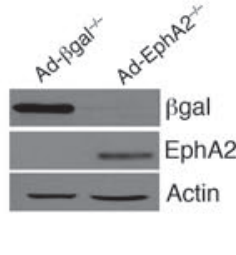

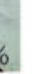

(1)
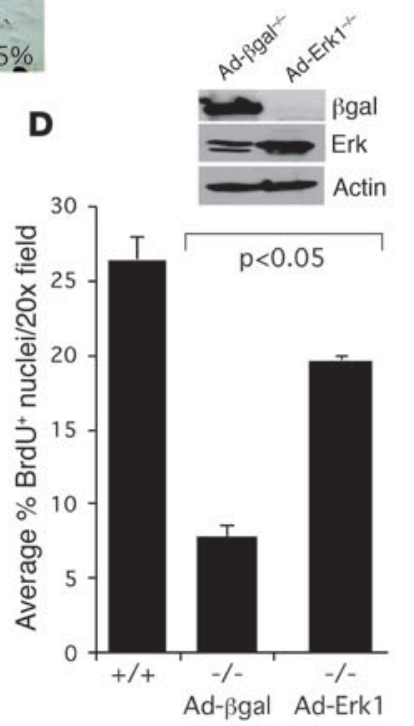

B

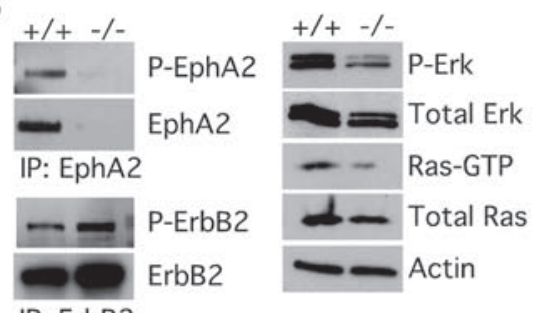

IP: ErbB2

\section{Figure 4}

EphA2 is required for Ras/Erk activation and proliferation in the context of Neu/ErbB2-mediated neoplasia. (A) Proliferation of PMTCs isolated from EphA2 $2^{--}$animals, as assessed by nuclear incorporation of BrdU (arrowheads), was reduced relative to EphA2 $2^{++}$cells. For rescue experiments, PMTCs were transduced with adenoviruses expressing EphA2 or $\beta$-gal 48 hours prior to BrdU incorporation assay. Overexpression of EphA2 significantly elevated serum-induced proliferation relative to control $(P<0.05 ; 2$-tailed, paired Student's $t$ test). Scale bar: $20 \mu \mathrm{m}$. Expression of adenoviral transgenes was confirmed by immunoblot. (B) Ras activity in unstimulated cells, as measured by effector pulldown assay of GTP-bound Ras by GST-Raf Ras-binding domain, was reduced in EphA2-- PMTCs relative to control, as was Erk phosphorylation. Uniform loading was confirmed by immunoblotting for total Ras/Erk and actin. EphA2 deficiency and uniform expression of Neu/ErbB2 was confirmed by effector pulldown assay and immunoblotting for EphA2 and ErbB2. EphA2 was phosphorylated in unstimulated EphA2 ${ }^{+/ /}$tumor cells, and no changes in ErbB2 phosphorylation were detected in EphA2+/+ versus EphA2 ${ }^{-/}$PMTCs. (C) Diminished Ras and Erk activity were confirmed in whole tumor extracts isolated from 3 independent EphA2 $2^{+/+}$or EphA2 $2^{--}$tumors. (D) For rescue experiments EphA2 $2^{--}$PMTCs were transduced with adenoviruses expressing Erk-1 or control $\beta$ gal. Overexpression of Erk-1 in EphA2-- PMTCs significantly elevated serum-induced proliferation relative to control $\left(P<0.05\right.$, EphA2 $2^{-/}$Ad- $\beta$-gal versus EphA2 $2^{+/}$or EphA2 $2^{-/}$Ad-Erk-1; single-factor ANOVA). Expression of adenoviral transgenes was confirmed by immunoblot. (E) Treatment of EphA2++/ PMTCs with the MEK inhibitor U0126 for 12 hours significantly inhibited serum-induced proliferation relative to vehicle control $(P<0.05,5$ - and 10- $\mu \mathrm{M}$ U0126 versus vehicle). Inhibition of Erk phosphorylation by U0126 was confirmed by immunoblot.

invasive protrusions in acinar structures formed by MCF10A and MCF10A.HER2 cells upon overexpression of EphA2, as assessed by confocal microscopy (Figure 3B). Quantification of nuclear Ki67 revealed that overexpression of EphA2 in MCF10A and MCF10A. HER2 cells increases proliferation nearly 3-fold compared with levels observed in control cells (Figure 3B). Overexpression of HER2 in MCF10A.HER2 cells, as well as expression of adenoviral gene products, was confirmed by immunoblot (Figure 3). Increased p-Erk levels in cells overexpressing HER2, and to a greater extent cells overexpressing both HER2 and EphA2, correlated with increased levels of proliferation in culture (Figure 3C). These data suggest that EphA2 enhances mammary epithelial proliferation and invasion and augments growth and invasive properties induced by ErbB2/HER2 in human breast epithelial cells.

EphA2 promotes activation of Ras/MAPK and tumor cell proliferation. To examine the specific EphA2 signaling events intrinsic to the breast epithelial cells that regulate proliferation, we purified MMTV-Neu hyperplastic PMECs and primary mammary tumor cells (PMTCs) from EphA2 ${ }^{-/-}$and EphA2 $2^{+/}$mice. EphA2 $2^{-/-}$tumor cells expressed no detectable levels of EphA2, but ErbB2 expression or phosphorylation 


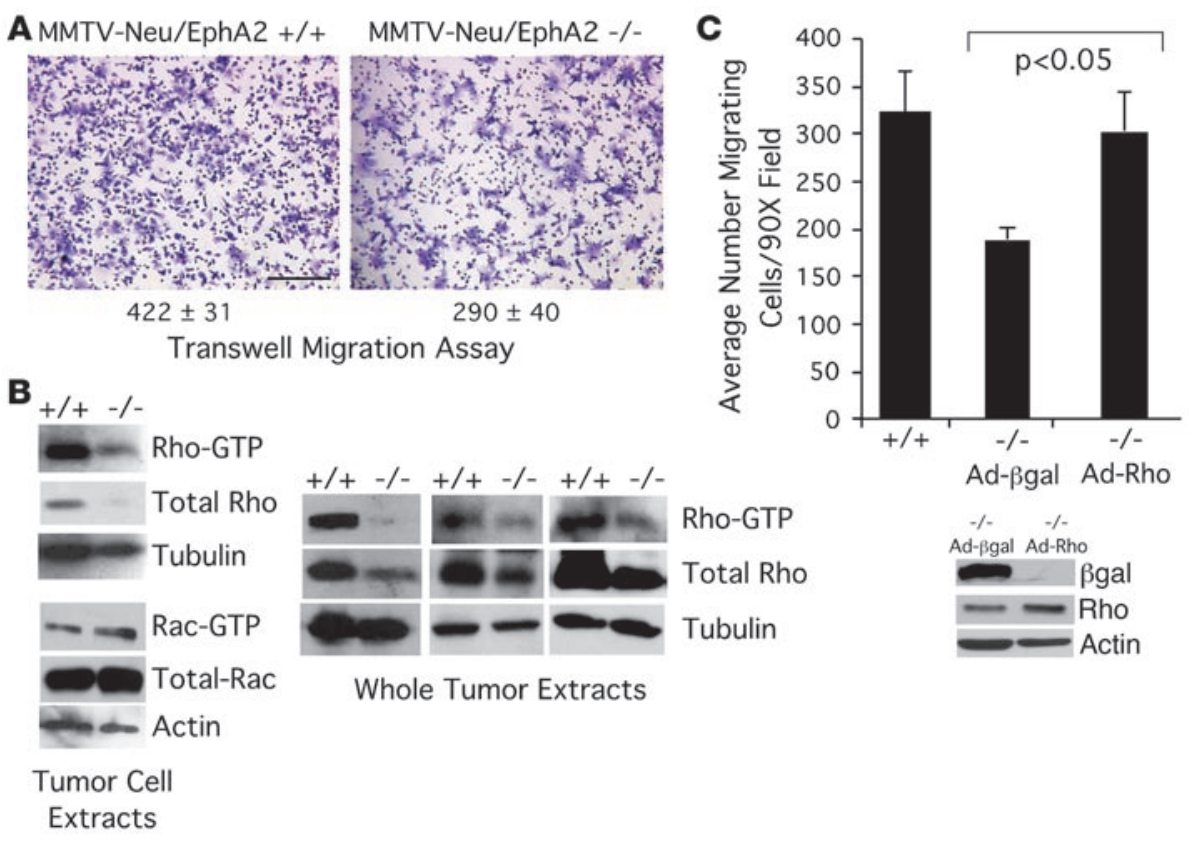

Figure 5

EphA2 is required for RhoA activation and tumor cell migration in the context of Neu/ErbB2-mediated malignancy. (A) EphA2 ${ }^{-1-}$ PMTCs displayed significantly reduced migration in response to growth media supplemented with $10 \%$ serum compared with EphA2 $2^{+/}$PMTCs in transwell migration assays ( $P<0.05$; 2-tailed, paired Student's $t$ test). (B) RhoA activity, as measured by effector pulldown assay of GTP-bound RhoA in tumor cell lysates and in whole tumor extracts by GST-Rhotekin Rho-binding domain, was reduced in $\mathrm{EphA}^{-/-} \mathrm{PMTC}$ and intact tumors relative to $\mathrm{EphA} 2^{+/+}$ cells and tumors. We also observed a decrease in total RhoA protein levels in EphA2-r- MMTV-Neu tumor cells and in whole tumor extracts relative to EphA2 ${ }^{+/+}$controls. We observed no change in GTP-bound, activated Rac, or total Rac protein levels in tumor cell lysates from EphA2 ${ }^{-/-}$or EphA2+/+ PMTCs. (C) For rescue experiments, EphA2-/- MMTV-Neu primary tumor cells were transduced with adenoviruses expressing constitutively active RhoA (Q63L) or control $\beta$-gal 48 hours prior to migration assay. Expression of constitutively active RhoA restored serum-induced migration of EphA2-l- tumor cells to levels comparable to those observed in tumor cells derived from EphA2 $2^{++}$animals, while control $\beta$-gal had no effect $\left(P<0.05\right.$, EphA2 ${ }^{-/-}$Ad- $\beta$-gal versus $\mathrm{EphA} 2^{+/+}$and $\mathrm{EphA} 2^{-/-} \mathrm{Ad}-\mathrm{Rho}$; single-factor ANOVA). Expression of adenoviral transgenes was confirmed by immunoblot assays.
EphA2 promotes tumor cell migration through activation of RhoA GTPase. To dissect the mechanisms by which EphA2 promotes tumor metastasis, we analyzed motility of MMTV-Neu tumor cells in the context of EphA2 deficiency using a transwell migration assay. EphA2-/MMTV-Neu tumor cells displayed a 1.5 -fold decrease in serum-stimulated migration relative to EphA2 ${ }^{+/+}$cells (Figure 5A). Because expression and activity of Rho family small GTPases are integral components of signaling pathways that regulate cell migration, we sought to determine whether EphA2 regulates tumor cell motility through a Rho-dependent mechanism. Diminished levels of active GTP-bound RhoA were present in both EphA2-/- tumors and in purified EphA2 $2^{-/}$PMTCs relative to EphA2 ${ }^{+/+}$controls (Figure 5B). EphA2 $2^{-/-}$tumor cells also displayed a decrease in total RhoA protein expression. In contrast, there were no detectable changes in levels of activated Rac1 under our experimental conditions. To determine whether activation of RhoA mediates EphA2-dependent cell migration, we expressed a constitutively active RhoA in EphA2-/- MMTV-Neu tumor cells. While expression from a control adenovirus expressing $\beta$-gal had no effect on migration in EphA2 $2^{-/}$PMTCs, expression of exogenous activated RhoA restored migration to levels similar to those of EphA2 $2^{+/+}$control cells (Figure 5C). These findings suggest that RhoA activation contributes to EphA2-mediated tumor cell migration.

While Rho family GTPases, includwas not affected in these cells (Figure 4B), suggesting that EphA2 did not regulate ErbB2 expression or activity. Both PMECs and PMTCs that were EphA2 $2^{--}$exhibited a decrease in proliferation relative to that in EphA2 ${ }^{+/+}$cells (Figure 4A and Figure 1D), and the proliferation defect was rescued by restoring EphA2 expression (Figure 4A). While there were no significant changes in levels of $\mathrm{p}$-src, p-stat5, p-cyclin-D1, or p-PLC $\gamma$ (data not shown), levels of p-Erk and active GTP-bound Ras were significantly diminished in EphA2 $2^{-/-}$relative to EphA2 ${ }^{+/+}$cells (Figure 4B and Supplemental Figure 3, A and B). Similarly, there was a substantial reduction in Erk and Ras activity in whole tumor lysates from EphA2-/- animals compared with tumors from EphA2 $2^{+/+}$MMTV-Neu mice (Figure 4C). Overexpression of exogenous Erk-1 rescued proliferation defects in EphA2-/- PMTCs relative to cells expressing control $\beta$-gal (Figure 4D), suggesting that modulation of Ras/Erk signaling is a primary mechanism through which EphA2 affects Neu-mediated tumor growth. Treatment of EphA2 ${ }^{+/+}$PMTCs with the MEK inhibitor U0126 significantly impaired proliferation. As MEK activates Erk in response to activation of Ras, these data confirm that Ras/MEK/Erk pathway activation regulates growth in these cells (Figure 4E). ing RhoA, have also been shown to regulate cell cycle progression $(35,36)$, expression of constitutively active RhoA did not rescue proliferation in EphA2/- PMTCs to the levels observed in control cells (data not shown), suggesting that RhoA activation specifically contributes to EphA2-mediated tumor cell migration rather than growth. Conversely, we did not observe any change in migration of EphA2-/- PMTCs upon overexpression of Erk-1 (data not shown). These data suggest that proliferation and motility are regulated separately by Erk-1 and Rho, respectively, in the context of ErbB2/EphA2-mediated tumor progression.

EphA2 physically and functionally interacts with ErbB2. To investigate the molecular mechanism(s) by which EphA2 modulates Neu/ ErbB2-mediated proliferation and invasiveness, biochemical studies were performed to assess physical interaction between EphA2 and ErbB2 in COS7 cells overexpressing both proteins and between endogenous proteins in MMTV-Neu-derived PMTCs. We detected the presence of ErbB2 in EphA2 immunoprecipitates, and EphA2 in ErbB2 immunoprecipitates, in lysates from COS7 cells overexpressing the human isoforms of EphA2 and ErbB2 (Figure 6A). Coimmunoprecipitation analysis of endogenous proteins from 

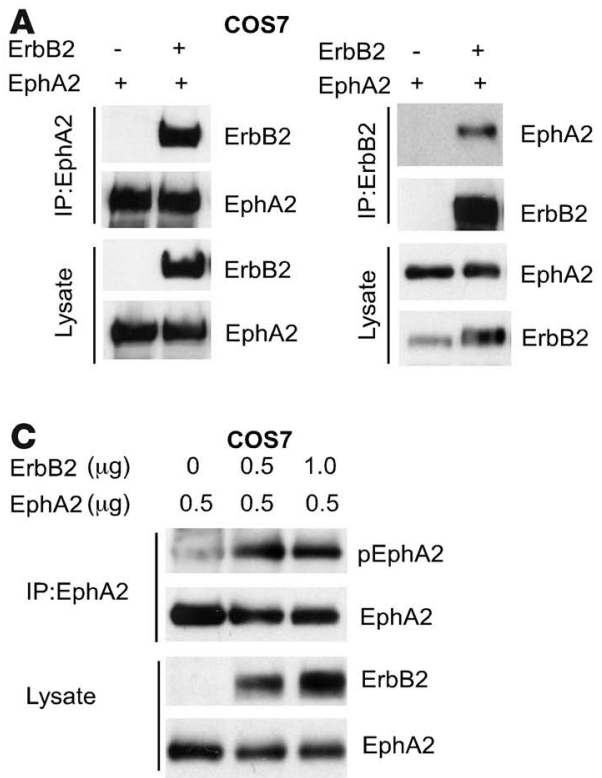
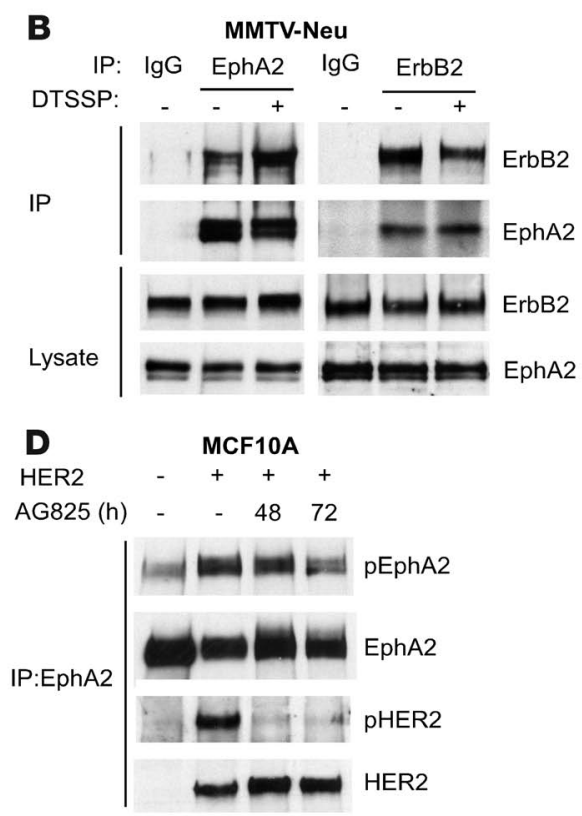

\section{Figure 6}

EphA2 physically and functionally interacts with ErbB2. (A) COS7 cells were transfected with plasmids for expression of EphA2 or/and ErbB2. EphA2 or ErbB2 was immunoprecipitated from cell lysates, and products were analyzed for ErbB2 or/and EphA2. Coexpression of EphA2 and ErbB2 was sufficient to permit coimmunoprecipitation. (B) Endogenous ErbB2 and EphA2 were coimmunoprecipitated with anti-EphA2 or anti-ErbB2 antibodies, respectively, in EphA2+/+ MMTV-Neu tumor cells that were untreated or treated with the chemical crosslinker DTSSP. The interaction detected was specific: EphA2 and ErbB2 were not immunoprecipitated by control IgG. Uniform input was validated by probing lysates for expression of EphA2 and ErbB2. (C) COS7 cells were transfected with plasmids for expression of EphA2 or ErbB2. EphA2 was immunoprecipitated from cell lysates, and products were analyzed for EphA2 expression and tyrosine phosphorylation. Coexpression of ErbB2 and EphA2 was sufficient to induce phosphorylation of EphA2 in COS7 cells in the absence of ephrin ligand stimulation. (D) Interaction between EphA2 and HER2 in MCF10A cells overexpressing HER2 was observed, as EphA2 and HER2 were coimmunoprecipitated with anti-EphA2 antibodies in HER2-overexpressing cells, but not in parental MCF10A cells. Elevated EphA2 phosphorylation was observed in MCF10A cells overexpressing HER2 relative to parental MCF10A cells, and treatment with the ErbB2 kinase inhibitor AG825 reduced EphA2 phosphorylation as well as ErbB2 phosphorylation in MCF10A cells overexpressing HER2.

PMTCs also confirmed that ErbB2 formed a complex with EphA2 (Figure 6B). In both PMTCs and COS7 cells, EphA2 and ErbB2 were expressed at high levels, and the EphA2/ErbB2 interaction occurred constitutively in the absence of ligand stimulation (Figure 6C). Strikingly, coexpression of ErbB2 and EphA2 in COS7 cells was sufficient to induce tyrosine phosphorylation of EphA2 in the absence of ligand or serum stimulation (Figure 6C). Likewise, elevated EphA2 phosphorylation was observed in MCF10A.HER2 cells overexpressing ErbB2 relative to parental MCF10A cells (Figure 6D). Consistent with coexpression data in COS7 cells, treatment with an ErbB2 kinase inhibitor diminished EphA2 phosphorylation as well as HER2 phosphorylation in MCF10A.HER2 cells (Figure 6D). Given evidence for physical interaction between ErbB2 and EphA2 and the functional requirement of EphA2 expression for maximal activation of signaling pathways downstream of ErbB2, these data suggest that that EphA2 participates in ErbB2 signaling.

EphA2 deficiency has no impact on tumor progression, angiogenesis, or metastasis in MMTV-PyV-mT transgenic animals. To assess EphA2 function in an independent endogenous model of mammary tumorigenesis that is also dependent upon the Ras/MAPK path-

Table 2 way, we crossed MMTV-PyV-mT mice with EphA2-/- mice. These animals were used to generate MMTV$\mathrm{PyV}-\mathrm{mT}$ mice that were EphA2 ${ }^{+/+}$, EphA2 ${ }^{+/-}$, or EphA2-/-. Virgin female mice were monitored for tumor formation through 100 days. Despite confirmed loss of EphA2 deficiency in the MMTV-PyV-mT model (Figure 7, A and C), EphA2 deficiency did not affect rate of tumor formation (data not shown), tumor volume, number of surface lung lesions, or microvascular density (Table 2 and Figure 7B). Additionally, there were no differences in levels of total Ras, active GTP-bound Ras, p-Erk, or total Rho in MMTV-PyV-mT tumors derived from EphA2 $2^{+/}$versus EphA2 $2^{-/}$mice (Figure 7C). These findings are in striking contrast to the effects of EphA2 deficiency observed in the MMTV-Neu model. These data suggest that, in marked contrast to the MMTV-Neu model, EphA2 does not affect tumor initiation, metastasis, or vascular density in the MMTV-PyV$\mathrm{mT}$ model, nor does loss of EphA2 affect the signaling pathways that contribute to these aspects of tumor progression in this model.

We next assessed expression and activation of EphA2 in normal mammary tissue isolated from FVB female mice, in MMTV-Neu and MMTVPyV-mT tumor tissue, and in PMECs and PMTCs isolated from both MMTV-Neu and MMTV-PyV-mT animals. EphA2 was overexpressed and phosphorylated in tumor tissue derived from both MMTV-Neu and MMTV-PyV-mT models compared with normal mammary tissue. Furthermore, expression of ephrin-A1 ligand was elevated in tumor lysates from both models compared with normal mammary tissue (Figure 7, C and D). Levels of ephrin-A1 were comparable in EphA2 ${ }^{+/+}$and EphA2-/- tumor lysates (Figure 7, C and D). Notably, however, levels of both total EphA2 and p-EphA2 were higher in MMTV-Neu tumors compared with MMTV-PyV-mT tumors (Figure 7D). EphA2 overexpression

Tumor volume and lung metastases in mice 100 days after birth

\begin{tabular}{|c|c|c|}
\hline Genotype & Tumor volume ${ }^{A}$ & No. lung lesions ${ }^{B}$ \\
\hline EphA2+/+ MMTV-PyV-mT & $747 \pm 291 \mathrm{~mm}^{3}$ & $10 \pm 6$ \\
\hline EphA2+/- MMTV-PyV-mT & $752 \pm 444 \mathrm{~mm}^{3}$ & $10 \pm 12$ \\
\hline EphA2-/- MMTV-PyV-mT & $803 \pm 329 \mathrm{~mm}^{3}$ & $12 \pm 7$ \\
\hline
\end{tabular}

Data are mean \pm SEM. $n=8-10$ per genotype; phenotypes were scored 100 days after birth. ${ }^{A} P=0.99$ among groups, single-factor ANOVA. ${ }^{B} P=0.32$ among groups, single-factor ANOVA. 


\section{A}

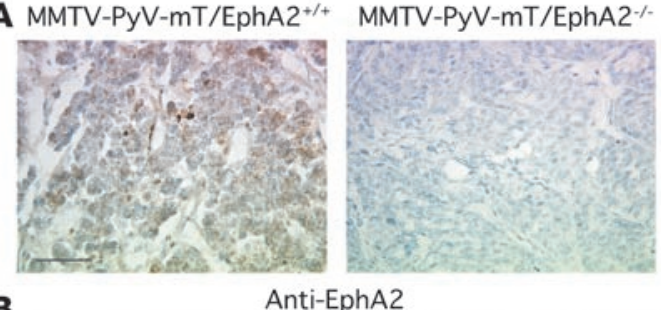

B
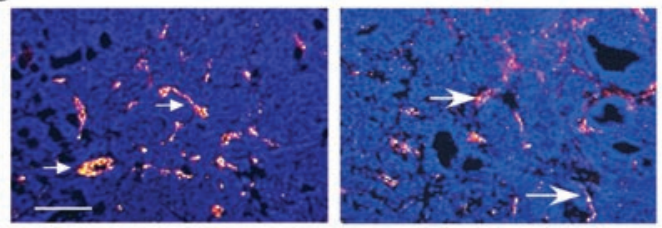

Anti-vWF

D
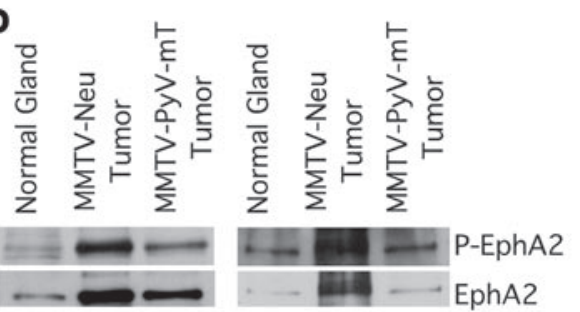

IP: EphA2

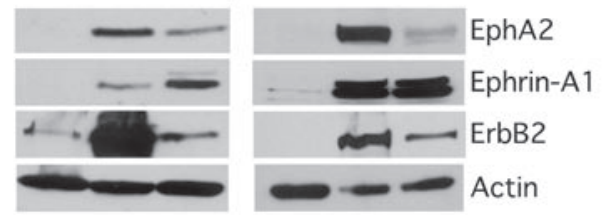

Whole Tissue Extracts

\section{C}

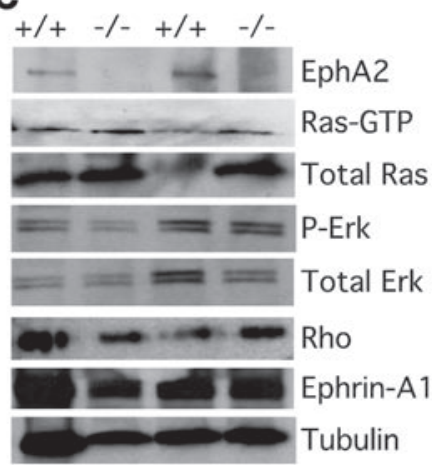

Whole Tumor Extracts
E

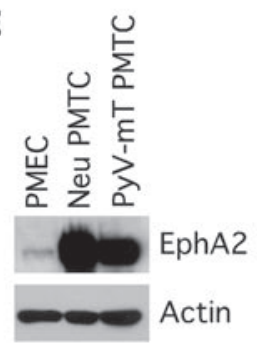

\section{Figure 7}

EphA2 deficiency does not affect tumorigenesis, microvascular density, or growth regulatory signaling pathways in MMTV-PyV-mT tumors. (A) Loss of EphA2 protein expression was confirmed by immunohistochemical staining. Scale bar: $50 \mu \mathrm{m}$. (B) We detected no change in MMTV-PyV-mT tumor microvascular density based on VWF staining (arrows indicate $\mathrm{VWF}^{+}$blood vessels). Scale bar: $100 \mu \mathrm{m}$. (C) We did not observe any change in levels of GTP-bound active Ras or p-Erk in EphA2 ${ }^{-/-}$MMTV-PyV-mT whole tumor extracts relative to controls, nor did we observe any change in levels of RhoA. Uniform loading was confirmed by immunoblotting for total Ras, total Erk, and tubulin. (D) We observed EphA2 overexpression and elevated phosphorylation in MMTV-Neu and MMTV-PyV-mT tumors relative to normal mammary tissue isolated from control FVB mice, with the highest levels observed in MMTV-Neu tumors. We also observed overexpression of ErbB2 and ephrin-A1 in both tumor types, with comparable ephrin-A1 expression in both tumor types and higher ErbB2 levels in MMTV-Neu tumors. Uniform loading was confirmed by immunoblot for actin. (E) We confirmed EphA2 overexpression specifically in epithelium by comparing EphA2 levels in PMEC lysates versus PMTCs derived from MMTV-Neu and MMTV-PyV-mT mice. was detected specifically in tumor cells and not in non-neoplastic epithelial cells (Figure 7E). While ErbB2 overexpression has been previously reported in MMTV-PyV-mT tumors (37) and was also observed in our tumor lysates, MMTV-Neu tumors displayed a much higher level of ErbB2 overexpression (Figure 7D). The evidence therefore suggests that EphA2 augments ErbB2/Neu-mediated signaling and that increased expression of EphA2 may be a mechanism by which ErbB2 signaling pathways are amplified in tumors.

Anti-EphA2 therapy shows efficacy in the MMTV-Nen tumor model. To determine whether MMTV-Neu tumors are responsive to targeted anti-EphA2 therapy in vivo, we transplanted wild-type MMTV-Neu tumor cells into the cleared fat pads of wild-type FVB recipient animals. At 2 weeks after transplantation, animals were injected intraperitoneally twice weekly for 3 weeks with either control IgG or an anti-EphA2 antibody that targets murine EphA2 for degradation (Figure 8A). The anti-EphA2 antibody specifically targeted EphA2, as expression of the related receptor EphA4 was unaffected in antibody-treated tumor cells derived from MMTV-Neu and MMTV-PyV-mT animals (Figure 8A). MMTV-Neu tumors harvested from anti-EphA2-treated animals displayed a 3-fold reduction in tumor volume relative to tumors isolated from IgG-treated mice (Figure 8B). In addition, tumor cell proliferation was significantly decreased in anti-EphA2-treated animals relative to controls, as determined by quantifying nuclear PCNA staining (Figure 8C). As predicted, EphA2 protein levels were significantly reduced in antiEphA2-treated tumors relative to control IgG-treated tumors, as assessed by immunohistochemistry and immunoblot (Figure 8D), although downregulation of EphA2 expression did not affect expression of ErbB2 in anti-EphA2-treated tumors, nor did control IgG treatment affect ErbB2 expression in tumors (Supplemental Figure 4A). We also observed a significant reduction in microvascular density in tumors harvested from anti-EphA2-treated animals relative to those treated with control IgG (Figure 8E). In contrast to these results, anti-EphA2 treatment had no effect on tumor volume (Figure 8F) or microvascular density (Supplemental Figure 4B) in animals transplanted with MMTV-PyV-mT tumors in spite of downregulated levels of EphA2 protein in anti-EphA2treated tumors (Supplemental Figure 4C). These data suggest that the efficacy of anti-EphA2 therapy depends upon the oncogene context in which tumor progression occurs, as treatment of MMTV-PyV-mT tumor-bearing animals did not affect tumor progression as in MMTV-Neu tumor-bearing mice in spite of EphA2 overexpression in both tumor models.

\section{Discussion}

Role of EphA2 in ErbB2-driven mammary tumor initiation and metastatic progression. The role of EphA2 in breast tumor progression has remained controversial, based on discrepancies among individual studies. For example, recent screens of RTK expression in tumors revealed that EphA2 RTK is overexpressed in a variety of human epithelial cancers, including more than $80 \%$ of breast cancer clinical samples $(38,39)$. While these studies did not discriminate between 

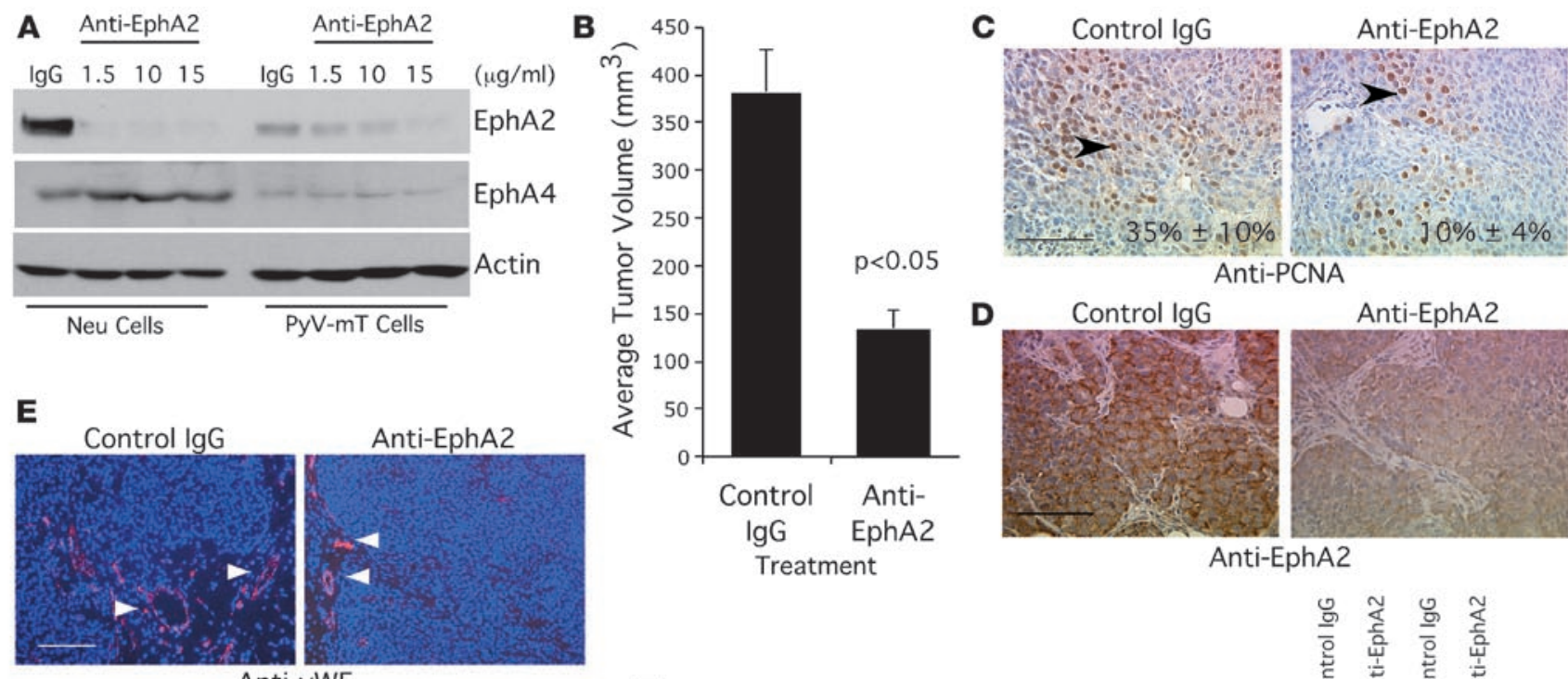

\section{Anti-PCNA}

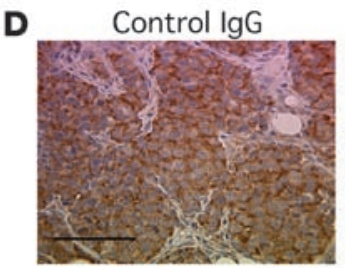

Anti-EphA2

Anti-EphA2
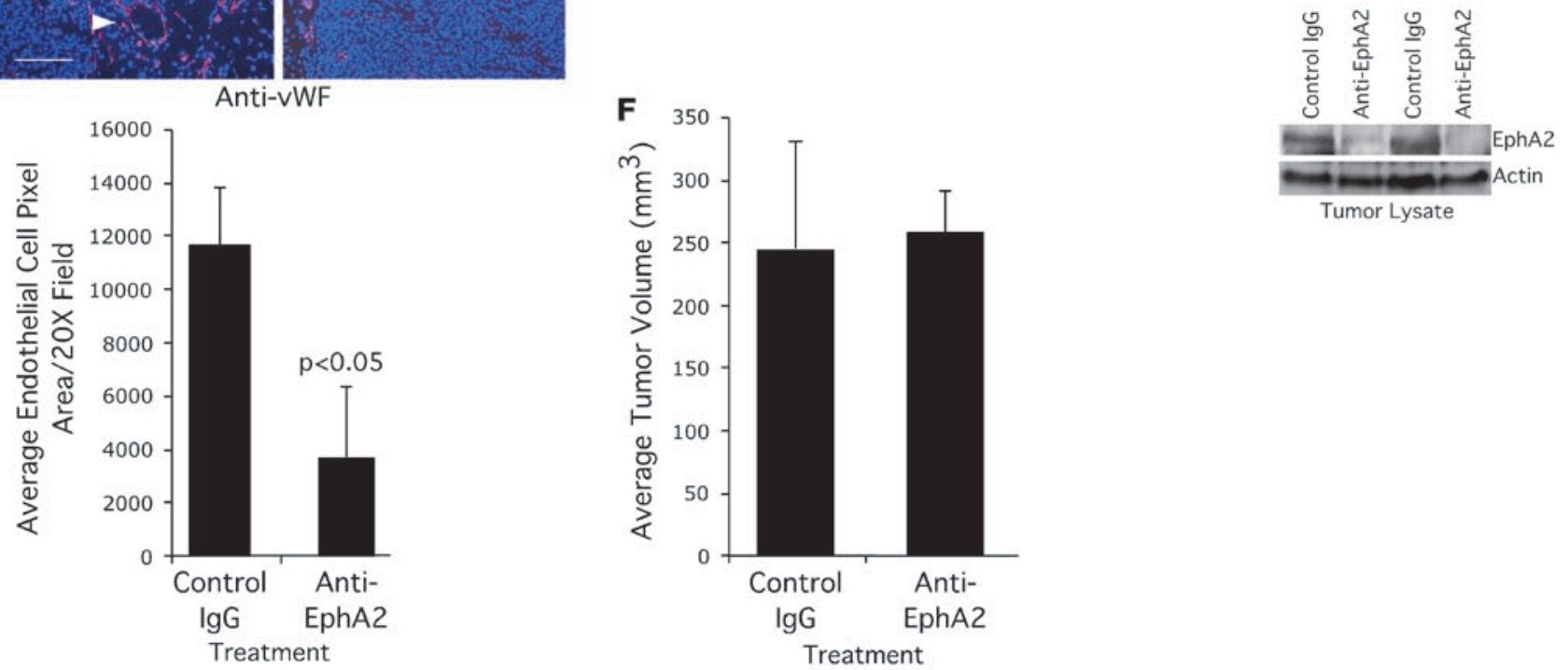

Figure 8

Treatment with an anti-EphA2 antibody inhibits tumor growth in MMTV-Neu but not MMTV-PyV-mT tumors. (A) Treatment with anti-murine EphA2 antibody diminished EphA2 protein expression in tumor cells derived from MMTV-Neu and MMTV-PyV-mT mice. Tumor cells were treated with control lgG $(10 \mu \mathrm{g} / \mathrm{ml})$ or increasing concentrations of anti-EphA2 antibody for 48 hours. Uniform loading was confirmed by immunoblot for actin. Blots were stripped and reprobed with anti-EphA4 antibodies as a control for antibody specificity. (B) Cells derived from EphA2 ${ }^{+/+}$ MMTV-Neu mice were orthotopically transplanted into the cleared fat pads of female FVB recipient mice. At 2 weeks following transplantation, mice were injected intraperitoneally with anti-EphA2 antibody or control lgG (10 mg/kg) twice weekly for 3 weeks. We observed a significant reduction in tumor volume in anti-EphA2-treated animals relative to control IgG-treated mice $(P<0.05$; 2-tailed, paired Student's $t$ test). Data are mean \pm SEM. (C) Tumor cell proliferation was significantly impaired in anti-EphA2-treated animals relative to controls $(P<0.05$; single-factor ANOVA; arrowheads indicate PCNA+ nuclei). Scale bar: $50 \mu \mathrm{m}$. (D) EphA2 expression was significantly diminished in anti-EphA2-treated tumors relative to IgG controls, as assessed by immunohistochemistry and immunoblot. Blots were stripped and reprobed for actin expression to verify uniform loading. Scale bar: $50 \mu \mathrm{m}$. (E) We observed significantly reduced $(P<0.05$; 2-tailed, paired Student's $t$ test) microvascular density in tumors isolated from anti-EphA2-treated mice relative to controls (arrowheads indicate vWF ${ }^{+}$blood vessels). Scale bar: $100 \mu \mathrm{m}$. (F) Cells derived from MMTV-PyV-mT mice were orthotopically transplanted in the cleared fat pad of FVB female recipient mice and were treated with anti-EphA2 antibody or control IgG as described above. We observed no change in tumor volume between animals treated with anti-EphA2 antibody relative to control IgG-treated mice.

stromal cell-intrinsic versus tumor cell-intrinsic EphA2 expression, they clearly demonstrated a correlation between EphA2 expression and breast cancer progression. In contrast, some initial studies suggested that EphA2 does not play a role in tumor initiation, including reports indicating that stimulation of tumor cells with soluble ephrins does not promote, and may even inhibit, MAPK activation and tumor cell proliferation (22-24). In addition, the majority of previous in vivo studies regarding EphA2 function in tumor progression involved tumor xenograft models that do not recapitulate endogenous tumor initiation and progression $(12-14,16,17,19,40,41)$. To provide a comprehensive examination of the role of EphA2 in the multiple stages of mammary tumorigenesis, we generated EphA2-/MMTV-Neu mice. We chose the MMTV-Neu model because the mammary epithelium progresses from hyperplasia to carcinoma in situ and to invasive and metastatic carcinoma in a stepwise manner is similar to that seen in ErbB2-overexpressing human breast 
cancers (30). EphA2 ${ }^{-/-}$MMTV-Neu female mice exhibited a reduction in mammary epithelial hyperplasia and developed tumors with reduced frequency secondary to a significant reduction in epithelial cell proliferation (Figure 1), consistent with a role for EphA2 in the earliest stages of breast cancer formation. This proliferation defect was, at least in part, intrinsic to epithelial cells, as BrdU incorporation was also reduced in primary mammary epithelial cells and tumor cells isolated from EphA2-/- animals relative to controls (Figures 1 and 4). Taken together, these results suggest that EphA2 is required for mammary tumor onset and growth.

At later stages of tumor progression, MMTV-Neu mammary tumors progress from hyperplasia to metastatic carcinoma through multiple steps, including increased tumor cell invasion and migration and sustained angiogenesis. Loss of EphA2 significantly inhibited MMTV-Neu tumor metastasis to the lung. While EphA2-dependent tumor cell proliferation is intrinsic to the epithelium, EphA2-dependent tumor metastasis can be attributed to dual roles for EphA2 in both tumor cells and host microenvironment. Ex vivo studies using EphA2-/- MMTV-Neu tumor cells revealed that loss of EphA2 impaired serum-induced cell migration (Figure 5), indicating a tumor cell-intrinsic role for EphA2-mediated malignant progression. Additionally, tumor angiogenesis was significantly diminished when MMTV-Neu cells were transplanted into EphA2-/- host environment (Supplemental Figure 2). These studies underscore the complex nature of EphA2 signaling.

Oncogene interaction in mammary tumorigenesis and metastatic progression. A large body of work indicates that tumorigenesis is a multistep process, and different oncogenes often cooperate to promote different steps of tumor progression (reviewed in refs. 1-3). Here we demonstrated a physical interaction between EphA2 and ErbB2 at the tumor cell surface, inducing phosphorylation of the EphA2 in the absence of ligand stimulation. This interaction between ErbB2 and EphA2 amplified Ras/Erk signaling and Rho GTPase activation (Figures 4 and 5), likely contributing to the increased proliferation and motility of EphA2-expressing tumor cells. This observation holds repercussions regarding how ErbB2-expressing breast cancers are treated, especially those that are refractory to anti-ErbB2 therapies. Our findings provide translational evidence that anti-EphA2 therapy may be effective against ErbB2-expressing tumors, alone or in combination with methods targeting ErbB2.

By contrast, EphA2 deficiency in MMTV-PyV-mT transgenic mice had no effect on tumor progression or in observed levels of activated Ras or p-Erk. Because Ras and Erk displayed high basal activity in $\mathrm{PyV}$-mT-expressing tumors regardless of EphA2 expression, these data suggest that $\mathrm{PyV}-\mathrm{mT}$ antigen activates Ras/MAPK pathway by alternate mechanisms, bypassing the requirement for EphA2 function. More importantly, this observation demonstrates that EphA2 function in tumor progression depends upon the context of other oncogenic/tumor suppressive determinants of malignancy, which may underlie some of the controversy regarding the role of EphA2 in tumor progression. While ErbB2 and PyV-mT activate many of the same signaling pathways that contribute to malignancy, such as those mediated by Ras, PI3K, src-family kinases, and Stat transcription factors (reviewed in refs. 42, 43), our data indicate that modulation of EphA2 signaling represents an important molecular distinction between these pathways to malignancy.

EphA2 RTK: oncogene or tumor suppressor? The contradictory observations that EphA2 may promote tumorigenesis under some circumstances (e.g., ErbB2-expressing breast tumors) and fail to influence tumor progression under others (e.g., PyV-mT- expressing tumors), or may even prevent tumor formation (e.g., carcinogen-induced skin cancers; ref. 22), may be reconciled by the following model that we propose. Under physiologic conditions, epithelial cells form adherens junction, permitting ephrins to interact with EphA2s on adjacent cells. Ligand stimulation induces receptor endocytosis and degradation, keeping EphA2 levels low. Upon tumor initiation, EphA2 expression is upregulated. Elevated EphA2 can be phosphorylated by other RTKs, such as ErbB2, independent of ligand stimulation, leading to enhanced cell proliferation and tumorigenesis. Thus, ligand stimulation may play an antitumorigenic role by downregulation of EphA2, while in the absence of ligand, EphA2 crosstalk with other receptors may promote tumorigenesis. This model is supported by several lines of experimental evidence. First, ligand stimulation has been shown to downregulate EphA2 through endocytosis $(24,44,45)$ as well as Cbl ubiquitin ligase-mediated proteasome degradation $(46,47)$. Second, adenoviral delivery of ephrin-A1 (45) and EphA2 activating antibodies have been shown to be effective in inhibition of malignant cell behavior in vitro and in treating malignant ovarian tumors in vivo $(48,49)$. Third, we have shown that ErbB-2 interacted with EphA2 physically in MMTV-Neu tumor cells and that EphA2 was phosphorylated by activated ErbB2. Fourth, ablation of EphA2 by gene targeting inhibited ErbB2-induced mammary tumorigenesis. Thus, EphA2 remains an important therapeutic target, and downregulation of EphA2 expression or inhibition of EphA2 signaling could lead to tumor inhibition.

EphA2 as a therapeutic target. Although EphA2 is overexpressed in a wide variety of tumors, including breast adenocarcinomas (reviewed in refs. 5-7), our data suggest that overexpression in and of itself does not necessarily indicate an active role in tumorigenesis. Significant levels of EphA2 overexpression were documented in tumors arising in both MMTV-Neu and MMTV-PyV-mT models of mammary carcinogenesis in this study. However, while deletion of EphA2 significantly impaired tumor initiation and progression in MMTV-Neu animals, there was no effect of EphA2 deficiency on tumor progression in the MMTV-PyV-mT model, which expressed only moderate levels of ErbB2. Thus, the functional consequences of EphA2 overexpression depend upon the context of coexpressed oncogenes. Therefore, effective therapeutic targeting of EphA2 requires an understanding of how EphA2 cooperates with and functionally influences coexisting oncogenic signaling networks within specific tumor types. For example, while downregulation of EphA2 protein levels showed efficacy against human ovarian tumor xenografts (49), an independent, similarly designed antibody reagent had no effect on CT26 human colon cancer xenografts or human mammary adenocarcinoma xenografts (50). Interestingly, like MMTV-PyV-mT tumor cells, CT26 cells do not overexpress ErbB2/HER2 (51), suggesting that EphA2 overexpression enhances malignant transformation and progression particularly in the context of ErbB2 overexpression and is therefore an appropriate target in such tumors.

While EphA2 overexpression has been reported in a variety of human epithelial cancers, including more than $80 \%$ of breast cancer clinical samples $(12,38,39)$, HER2 overexpression is observed in only $30 \%$ of human breast cancers (52). Moreover, no correlation was reported between EphA2 and HER2 expression in a recent screen of 134 human breast cancer specimens (39). Our data demonstrated that EphA2 interacted with ErbB2. Other EGFR family members, including EGFR/ErbB1 and an EGFR variant (EGFRvIII, a constitutively active deletion mutant implicated in carcinogen- 
esis; refs. 53, 54), have also been shown to physically and functionally interact EphA2 (55). Overexpression of EGFR and EGFRvIII has been reported in a broader subset of human breast cancers, with as many as $48 \%$ of cases analyzed reported to be positive for EGFR expression (56-61). Thus, EphA2 may act in concert with the EGFR family of RTKs in general, and not exclusively with ErbB2, to enhance proliferation and malignant progression. Functional interaction between EphA2 and EGFR as well as ErbB2 may be required for breast tumor growth and progression. Further investigation of the relationship between EphA2 and EGFR family members may provide a sound rationale for targeting EphA2 in breast cancers that display amplification and/or activation of 1 or more of this family of RTKs.

Conclusions. Our data suggest that the role of EphA2 RTK in cancer is context dependent, as EphA2 deficiency impairs tumor progression in MMTV-Neu, but not MMTV-PyV-mT, transgenic models of mammary epithelial adenocarcinoma. We provide evidence that EphA2 physically and functionally interacts with ErbB2 to amplify Ras/MAPK and RhoA signaling in tumor cells. Ras/MAPK contributes to cell proliferation, while activated Rho GTPase is required for tumor cell motility. Together, these results indicate that EphA2 cooperates with ErbB2/Neu to promote tumor progression and may be a novel target for tumors that are dependent upon ErbB receptor signaling.

\section{Methods}

Reagents. Antibodies against the following proteins were used: EphA2 (Zymed Laboratories, Santa Cruz Biotechnology, and Upstate Biotechnology); EphA4 (Upstate Biotechnology); PCNA (BD Biosciences); anti-Erk, anti-phosphothreonine-202/tyrosine-204 Erk, Akt, and phosphoserine473 Akt (Cell Signaling Technology); anti-tubulin (Sigma-Aldrich); ErbB2 (Neomarkers/Lab Vision Corporation); anti- $\beta$-actin (Santa Cruz Biotechnology); Ras (BD Biosciences); RhoA (Santa Cruz Biotechnology and BD Biosciences); vWF (Zymed Laboratories); E-cadherin (BD Biosciences); Ki67 (Vision Biosystems Inc.); and normal rabbit IgG (Santa Cruz Biotechnology). Therapeutic anti-EphA2 (1C1) and control nonspecific IgG (R347) antibodies were provided by MedImmune Inc. Raf-1 RBD agarose Ras assay reagent was purchased from Upstate Biotechnology. BrdU was purchased from Sigma-Aldrich. BrdU detection and ApopTag Red In situ Apoptosis kits were purchased from Zymed Laboratories and Chemicon/Millipore, respectively. Avidin peroxidase reagents were from Vector Laboratories, and liquid 3,3'-diaminobenzidine tetrahydrochloride (DAB) substrate kit was from Zymed Laboratories. Ephrin-A1-Fc was from R\&D Systems. Estrogen, progesterone, insulin, and EGF were from Sigma-Aldrich. DAPI was purchased from Sigma-Aldrich. TO-PRO-3 iodide nuclear stain, CellTracker orange CMTMR, and CellTracker green CMFDA dyes was purchased from Invitrogen. Growth factor-reduced Matrigel was purchased from BD Biosciences. AG825 ErbB2 kinase inhibitor was from Calbiochem (EMD Biosciences). Recombinant adenoviruses expressing constitutively active RhoA (Q63L) and Erk-1 were purchased from Cell Biolabs and Vector Biolabs, respectively. Control adenoviruses expressing $\beta$-gal and adenoviruses expressing EphA2 have been previously described $(62,63)$. MEK inhibitor U0126 was purchased from Calbiochem.

Mice and in vivo tumor studies. All animals were housed under pathogenfree conditions, and experiments were performed in accordance with AAALAC guidelines and with Vanderbilt University Institutional Animal Care and Use Committee approval. EphA2 $2^{-/}$mice were backcrossed with FVB animals for 5-7 generations prior to crossing with MMTV-Neu or MMTV-PyV-mT mice on an inbred FVB background (Jackson Laboratories; refs. 29, 30). MMTV-Neu- or MMTV-PyV-mT-positive transgenic animals that were EphA2 $2^{+/+}$EphA2 $2^{+/}$, or EphA2 $2^{--}$(63) were identified by PCR analysis of genomic DNA from tail biopsy using the following primers: 5'-GGGTGCCAAAGTAGAACTGCG-3' (forward), 5'-GACAGAATAAAACGCACGGGTG-3' (neo), 5'-TTCAGCCAAGCCTATGTAGAAAGC-3' (reverse). The neu and $P y V-m T$ transgenes were detected by PCR using primers and conditions recommended by Jackson Laboratories. Age-matched littermates were monitored for tumor formation by weekly palpation.

Tumors and lungs were collected from 2 cohorts of MMTV-Neu hemizygous $\mathrm{EphA} 2^{+/+}, \mathrm{EphA2} 2^{+/}$, and EphA2-/- animals at 8 months and 1 year after birth. Tumors and lungs were collected from MMTV-PyV-mT hemizygous EphA1 ${ }^{+/}$, EphA1 ${ }^{+/}$, and EphA1 $1^{-/-}$animals 100 days after birth. Tumors were enumerated, and dimensions were measured by caliper. Tumor volume was calculated as $l \times w^{2} \times 0.52$, where $l$ represents length and $w$ width (64). Lungs were fixed and dehydrated, and surface metastases were enumerated. For transplantation studies, the left inguinal mammary gland fat pad of 3-week-old recipient EphA2 ${ }^{+/+}$or EphA2 /- FVB female animals was cleared of endogenous epithelium as described previously (65) and injected with $10^{6}$ tumor cells derived from MMTV-Neu (31) or MMTV-PyV-mT (66) animals. Resulting tumors were harvested 4-5 weeks after injection for analysis. Where indicated, beginning at 2 weeks after tumor cell injection, recipient mice received intraperitoneal injections of $1 \mathrm{C} 1$ anti-EphA2 antibody or control IgG (10 mg/kg twice weekly for 3 weeks) prior to collection and analysis of primary tumors. At least 10 animals per condition were analyzed in 2-3 independent experiments. Statistical analysis of tumor development and metastasis frequency was assessed by $\chi^{2}$ test assuming that $50 \%$ of MMTV-Neu female mice should develop tumors within 7-8 months after birth, as was originally reported (30).

Histologic analyses. Mammary glands and tumors were harvested at the indicated time points and fixed in 10\% neutral buffered formalin (Fisher Scientific) for 24 hours at $4^{\circ} \mathrm{C}$. Whole-mount hematoxylin staining of mammary glands and $\mathrm{H} \& \mathrm{E}$ staining of $7-\mu \mathrm{m}$ mammary gland tissue sections was performed as described previously (65). Immunohistochemical staining for EphA2 and PCNA was performed as described previously (17), and proliferation was quantified by calculating the average percentage of $\mathrm{PCNA}^{+}$nuclei relative to total nuclei ( 4 random fields of at least 4 independent mammary and tumor samples per genotype; original magnification, $\times 20$ ). Apoptosis assays were performed using the Apoptag red in situ apoptosis detection kit per the manufacturer's protocol (Chemicon International). Apoptosis was calculated as the average percentage $\mathrm{TUNEL}^{+}$nuclei relative to total nuclei ( 4 random fields of at least 4 independent mammary and tumor samples per genotype; original magnification, $\times 20$ ). We detected p-Erk in tissue sections using rabbit monoclonal anti-p-Erk antibody clone 20G11 per the manufacturer's protocol (Cell Signaling Technology). Colorimetric immunohistochemical staining for $\mathrm{vWF}$ was performed by the Vanderbilt University Immunohistochemistry Core Facility, and immunofluorescence staining was performed as described previously (8). Microvascular density was determined by counting the number of $\mathrm{vWF}^{+}$vessels in 4 random fields per sample of at least 4 independent tumors per genotype (original magnification, $\times 20$ ). ErbB2 immunohistochemistry was performed using $5 \mu \mathrm{g} / \mathrm{ml}$ rabbit anti-ErbB2 antibody (Neomarkers/Lab Vision Corporation).

Cell culture. PMECs were isolated from mice as described previously (65, 67) and maintained in PMEC media (DMEM/F12 media [Mediatech] supplemented with $5 \mathrm{ng} / \mathrm{ml}$ estrogen, $5 \mathrm{ng} / \mathrm{ml}$ progesterone, $5 \mathrm{ng} / \mathrm{ml} \mathrm{EGF}$, and $5 \mu \mathrm{g} / \mathrm{ml}$ insulin [Sigma-Aldrich]) on growth factor-reduced Matrigel-coated (1:20 dilution) tissue culture dishes. Primary tumor cells were derived from EphA2 $2^{+/+}$or EphA2-/- MMTV-Neu animals as previously described (31). Enrichment of tumor cells in cultures was verified by expression of the neu transgene (31). The MMTV-Neu tumor-derived cell line (31) and the MMTV-PyV-mT tumor-derived cell line (66) used in transplantation and signaling studies were cultured in PMEC media. For EphA2 degradation 
studies, tumor cells were cultured in the presence of 1C1 anti-EphA2 antibody or control IgG (MedImmune) at the indicated concentrations for 48 hours prior to harvesting lysates for immunoblot analysis. In vitro proliferation and apoptosis analyses were performed as described previously $(17,67)$ using BrdU and TUNEL detection kits described above. For rescue experiments, EphA2-/- MMTV-Neu primary tumor cells were transduced with $1 \times 10^{8} \mathrm{pfu} / \mathrm{ml}$ adenovirus expressing Erk-1, EphA2, or control $\beta$-gal 48 hours prior to BrdU assay. For MEK inhibitor studies, cells were treated with 5 and $10 \mu \mathrm{M} \mathrm{U} 0126$ (Calbiochem) or DMSO vehicle control for the 12 hours during the BrdU labeling/serum stimulation time frame. Transwell migration assays were performed as described previously (63). For rescue experiments, EphA2 -/- MMTV-Neu primary tumor cells were transduced with $1 \times 10^{8} \mathrm{pfu} / \mathrm{ml}$ adenovirus expressing constitutively active RhoA (Q63L) or control $\beta$-gal 48 hours prior to transwell assay. Tumor-endothelial cell coculture migration assays were performed as described previously $(8,68)$.

siRNA sequences for mouse EphA2 or irrelevant control sequences were cloned into pRetroSuper viral vector and used to produce retroviruses for infection of MMTV-Neu tumor cells as previously described $(68,69)$. The following sequences were used to target EphA2: siRNA no. 1, 5'-GCCAAAGTAGAACTGCGTT-3' (aa 1,140-1,158); siRNA no. 2, 5'-GCGCTAGACAAGTTCCTTA-3' (aa 2,211-2,229); control siRNA, 5'-GCACCAGTTCAGCAAGACT-3'. We established 3-dimensional spheroid cultures as described previously (70). Cultures were maintained for 8 days prior to photodocumentation. Digital images were scored for spheroid culture area in 4 random fields, 3 cultures per field, using NIH ImageJ software. For confocal imaging, spheroid cultures were fixed in $10 \%$ neutral buffered formalin and subjected to immunohistochemistry for E-cadherin followed by nuclear staining with TO-PRO-3 as previously described (71). Tumor cells were transplanted into the cleared fat pads of recipient FVB mice as described above. At least 10 animals per condition were analyzed in 2-3 independent experiments.

Parental MCF10A and MCF10A cells stably overexpressing HER2 were maintained as described previously (34). We established 3-dimensional spheroid cultures as described previously (70). Cells were transduced with $1 \times 10^{8} \mathrm{pfu} / \mathrm{ml}$ adenovirus expressing constitutively EphA2 or control $\beta$-gal 48 hours prior to analysis. Staining for confocal analysis was performed as described above.

Immunoprecipitation and immunoblot analysis. Immunoprecipitation and immunoblot of EphA2 was performed as described previously (17). ErbB2 was immunoprecipitated using $1 \mu \mathrm{g}$ rabbit anti-ErbB2 plus $1 \mu \mathrm{g}$ mouse anti-ErbB2 Ab-17 (Neomarkers/Lab Vision Corporation). Where indicated, $2.5 \times 10^{5}$ PMECs (for Western analyses) or $2.5 \times 10^{6}$ primary tumor cells (for GTP-Ras and -Rho/Rac pulldown assays) were cultured in DMEM: F12 media plus 2\% FBS overnight. For analysis of EphA2 stability, MMTVNeu or MMTV-PyV-mT tumor cells $\left(2.5 \times 10^{6}\right)$ were treated with EphA2agonist monoclonal antibody $1 \mathrm{C} 1$ or control IgGs at the indicated doses and times. Lysates were harvested and used for immunoblot analysis as described previously (17). Densitometric analysis was performed using NIH ImageJ software.

1. Hanahan, D., and Weinberg, R.A. 2000. The hallmarks of cancer. Cell. 100:57-70.

2. Hahn, W.C., and Weinberg, R.A. 2002. Rules for making human tumor cells. N. Engl. J. Med. 347:1593-1603.

3. Vogelstein, B., and Kinzler, K.W. 2004. Cancer genes and the pathways they control. Nat. Med. 10:789-799.

4. Blume-Jensen, P., and Hunter, T. 2001. Oncogenic kinase signalling. Nature. 411:355-365.

5. Brantley-Sieders, D.M., and Chen, J. 2004. Eph receptor tyrosine kinases in angiogenesis: from development to disease. Angiogenesis. 7:17-28.

6. Brantley-Sieders, D., Parker, M., and Chen, J. 2004.

For Ras and Rho/Rac pulldown assays, tumor tissue was collected, weighed, mechanically homogenized in PBS, pelleted, and resuspended in manufacturer-recommended assay buffer (Upstate Biotechnology). Approximately $500 \mu \mathrm{g}$ tumor lysate was used per assay. Ras assays were performed using Raf-1 Ras-binding domain-GST assay reagent (Upstate Biotechnology) per the manufacturer's protocol. Rho assays were performed using Rhotekin-binding domain-GST reagents as previously described (16). For some coimmunoprecipitation assays, COS7 cells were cotransfected with $1 \mu \mathrm{g}$ each of myc-tagged erbB2 (pcDNA3-erbB2) and ephA2 (pcDNA3-EphA2) using Lipofectamine 2000 (Invitrogen). Cells were lysed in 1\% NP-40 buffer (10 mM Tris-HCl, pH 7.5; $150 \mathrm{mM} \mathrm{NaCl} ; 2 \mathrm{mM}$ EDTA; and $1 \%$ NP-40 plus $50 \mathrm{mM}$ protease inhibitors). Lysates were used for immunoprecipitation with anti-myc (Sigma-Aldrich) or anti-EphA2 antibodies (catalog no. sc-924; Santa Cruz). Immune complexes were resolved on SDS-PAGE and Western blotted using anti-EphA2 or anti-myc antibodies. EphA2 was immunoprecipitated from MMTV-Neu cells, followed by treatment of half the samples with the $1 \mathrm{mM}$ of the crosslinking agent DTSSP. Immunoprecipitates were subjected to Western blot analysis using anti-ErbB2 (1:2,000 dilution; Neomarkers). EphA2 and ErbB2 were immunoprecipitated from MCF10A and MCF10A.HER2 cells as described above. Where indicated, cells were incubated with $10 \mu \mathrm{g} / \mathrm{ml}$ AG825 ErbB2 kinase inhibitor for 24 or 48 hours prior to immunoprecipitation.

Statistics. Statistical differences among groups were determined by single-factor ANOVA, by 2-tailed, paired Student's $t$ test; or by $\chi^{2}$ test as indicated in the figure and table legends. A $P$ value less than 0.05 was considered significant.

\section{Acknowledgments}

This work was supported by NIH grants CA95004 and CA114301 (J. Chen), NIH grant CA1179151-02 (D. Brantley-Sieders), and Department of Defense predoctoral fellowship W81XWH-05-10254 (W.B. Fang). We thank Carlos Arteaga at Vanderbilt University for advice and helpful discussions and for providing cell lines derived from MMTV-Neu and MMTV-PyV-mT animals as well as parental and MCF10A.HER2-overexpressing cell lines. We also thank Alissa Weaver and Nichole Bryce at Vanderbilt University for protocols and advice on confocal image analysis of 3-dimensional spheroid cultures.

Received for publication July 2, 2007, and accepted in revised form October 17, 2007.

Address correspondence to: Jin Chen, Vanderbilt University School of Medicine, A-4323 MCN, 1161 21st Avenue South, Nashville, Tennessee 37232-2363, USA. Phone: (615) 343-3819; Fax: (615) 343-8648; E-mail: jin.chen@vanderbilt.edu.

Dana M. Brantley-Sieders and Guanglei Zhuang contributed equally to this work.

Eph receptor tyrosine kinases in tumor and tumor microenvironment. Curr. Pharm. Des. 10:3431-3442.

7. Ireton, R.C., and Chen, J. 2005. EphA2 receptor tyrosine kinase as a promising target for cancer therapeutics. Curr. Cancer Drug Targets. 5:149-157.

8. Brantley-Sieders, D.M., et al. 2005. Impaired tumor microenvironment in EphA2-deficient mice inhibits tumor angiogenesis and metastatic progression. FASEB J. 19:1884-1886

9. Murai, K.K., and Pasquale, E.B. 2003. 'Eph'ective signaling: forward, reverse and crosstalk. J. Cell Sci. 116:2823-2832.

10. Poliakov, A., Cotrina, M., and Wilkinson, D.G. 2004. Diverse roles of eph receptors and ephrins in the regulation of cell migration and tissue assembly. Dev. Cell. 7:465-480.

11. Pasquale, E.B. 2005. Eph receptor signalling casts a wide net on cell behaviour. Nat. Rev. Mol. Cell Biol. 6:462-475.

12. Zelinski, D.P., et al. 2001. EphA2 overexpression causes tumorigenesis of mammary epithelial cells. Cancer Res. 61:2301-2306.

13. Duxbury, M.S., Ito, H., Zinner, M.J., Ashley, S.W., and Whang, E.E. 2004. EphA2: a determinant of malignant cellular behavior and a potential therapeutic target in pancreatic adenocarcinoma. Oncogene. 23:1448-1456.

14. Landen, C.N., Jr., et al. 2005. Therapeutic EphA2 gene 
targeting in vivo using neutral liposomal small interfering RNA delivery. Cancer Res. 65:6910-6918.

15. Nasreen, N., Mohammed, K.A., and Antony, V.B. 2006. Silencing the receptor EphA2 suppresses the growth and haptotaxis of malignant mesothelioma cells. Cancer. 107:2425-2435.

16. Fang, W.B., Brantley-Sieders, D.M., Parker, M.A. Reith, A.D., and Chen, J. 2005. A kinase-dependent role for EphA2 receptor in promoting tumor growth and metastasis. Oncogene. 24:7859-7868.

17. Brantley, D.M., et al. 2002. Soluble Eph A receptors inhibit tumor angiogenesis and progression in vivo. Oncogene. 21:7011-7026

18. Cheng, N., et al. 2003. Inhibition of VEGF-dependent multistage carcinogenesis by soluble EphA receptors. Neoplasia. 5:445-456.

19. Dobrzanski, P., et al. 2004. Antiangiogenic and antitumor efficacy of EphA2 receptor antagonist. Cancer Res. 64:910-919.

20. Pratt, R.L., and Kinch, M.S. 2002. Activation of the EphA2 tyrosine kinase stimulates the MAP/ERK kinase signaling cascade. Oncogene. 21:7690-7699.

21. Pratt, R.L., and Kinch, M.S. 2003. Ligand binding up-regulates EphA2 messenger RNA through the mitogen-activated protein/extracellular signal-regulated kinase pathway. Mol. Cancer Res. 1:1070-1076.

22. Guo, H., et al. 2006. Disruption of EphA2 receptor tyrosine kinase leads to increased susceptibility to carcinogenesis in mouse skin. Cancer Res. 66:7050-7058

23. Miao, H., et al. 2001. Activation of EphA receptor tyrosine kinase inhibits the Ras/MAPK pathway. Nat. Cell Biol. 3:527-530.

24. Macrae, M., et al. 2005. A conditional feedback loop regulates Ras activity through EphA2. Cancer Cell. 8:111-118.

25. Dohn, M., Jiang, J., and Chen, X. 2001. Receptor tyrosine kinase EphA2 is regulated by p53-family proteins and induces apoptosis. Oncogene. 20:6503-6515.

26. Zhang, W., et al. 2003. Apoptotic response to 5 -fluorouracil treatment is mediated by reduced polyamines, non-autocrine Fas ligand and induced tumor necrosis factor receptor 2. Cancer Biol. Ther 2:572-578.

27. Yang, G., Zhang, G., Pittelkow, M.R., Ramoni, M., and Tsao, H. 2006. Expression Profiling of UVB Response in Melanocytes Identifies a Set of p53Target Genes. J. Invest. Dermatol. 126:2490-2506.

28. Jin, Y.J., et al. 2006. A novel mechanism for p53 to regulate its target gene ECK in signaling apoptosis Mol. Cancer Res. 4:769-778.

29. Guy, C.T., Cardiff, R.D., and Muller, W.J. 1992. Induction of mammary tumors by expression of polyomavirus middle $\mathrm{T}$ oncogene: a transgenic mouse model for metastatic disease. Mol. Cell. Biol. 12:954-961.

30. Guy, C.T., et al. 1992. Expression of the neu protooncogene in the mammary epithelium of transgenic mice induces metastatic disease. Proc. Natl. Acad. Sci. U. S. A. 89:10578-10582.

31. Muraoka, R.S., et al. 2003. Increased malignancy of Neu-induced mammary tumors overexpressing active transforming growth factor beta1. Mol. Cell. Biol. 23:8691-8703.

32. Eccles, S.A. 2001. The role of c-erbB-2/HER2/ neu in breast cancer progression and metastasis. J. Mammary Gland Biol. Neoplasia. 6:393-406.

33. Muthuswamy, S.K., Li, D., Lelievre, S., Bissell, M.J., and Brugge, J.S. 2001. ErbB2, but not ErbB1, reinitiates proliferation and induces luminal repopulation in epithelial acini. Nat. Cell Biol. 3:785-792.

34. Ueda, Y., Wang, S., Dumont, N., Yi, J.Y., Koh, Y., and Arteaga, C.L. 2004. Overexpression of HER2 (erbB2) in human breast epithelial cells unmasks transforming growth factor beta-induced cell motility. J. Biol. Chem. 279:24505-24513.

35. Olson, M.F., Ashworth, A., and Hall, A. 1995. An essential role for Rho, Rac, and Cdc42 GTPases in cell cycle progression through G1. Science. 269:1270-1272.

36. Welsh, C.F., et al. 2001. Timing of cyclin D1 expression within G1 phase is controlled by Rho. Nat. Cell Biol. 3:950-957.

37. Lin, E.Y., et al. 2003. Progression to malignancy in the polyoma middle $\mathrm{T}$ oncoprotein mouse breast cancer model provides a reliable model for human diseases. Am. J. Pathol. 163:2113-2126.

38. Ogawa, K., et al. 2000. The ephrin-A1 ligand and its receptor, EphA2, are expressed during tumor neovascularization. Oncogene. 19:6043-6052.

39. Pan, M. 2005. Overexpression of EphA2 gene in invasive human breast cancer and its association with hormone receptor status. Journal of Clinical Oncology, 2005 ASCO Annual Meeting Proceedings. 23(Suppl.):9583.

40. Lu, M., Miller, K.D., Gokmen-Polar, Y., Jeng, M.H., and Kinch, M.S. 2003. EphA2 overexpression decreases estrogen dependence and tamoxifen sensitivity. Cancer Res. 63:3425-3429.

41. Duxbury, M.S., Ito, H., Zinner, M.J., Ashley, S.W., and Whang, E.E. 2004. Ligation of EphA2 by Ephrin A1-Fc inhibits pancreatic adenocarcinoma cellular invasiveness. Biochem. Biophys. Res. Commun. 320:1096-1102.

42. Dankort, D.L., and Muller, W.J. 2000. Signal transduction in mammary tumorigenesis: a transgenic perspective. Oncogene. 19:1038-1044

43. Ichaso, N., and Dilworth, S.M. 2001. Cell transformation by the middle $\mathrm{T}$-antigen of polyoma virus. Oncogene. 20:7908-7916.

44. Zhuang, G., Hunter, S., Hwang, Y., and Chen, J. 2007. Regulation of EphA2 receptor endocytosis by SHIP2 lipid phosphatase via phosphatidylinosito 3-Kinase-dependent Rac1 activation. J. Biol. Chem. 282:2683-2694.

45. Noblitt, L.W., et al. 2004. Decreased tumorigenic potential of EphA2-overexpressing breast cancer cells following treatment with adenoviral vectors that express EphrinA1. Cancer Gene Ther. 11:757-766.

46. Walker-Daniels, J., Riese, D.J., 2nd, and Kinch, M.S 2002. c-Cbl-dependent EphA2 protein degradation is induced by ligand binding. Mol. Cancer Res. 1:79-87.

47. Wang, Y., et al. 2002. Negative regulation of EphA2 receptor by Cbl. Biochem. Biophys. Res. Commun. 296:214-220.

48. Carles-Kinch, K., Kilpatrick, K.E., Stewart, J.C., and Kinch, M.S. 2002. Antibody targeting of the EphA2 tyrosine kinase inhibits malignant cell behavior. Cancer Res. 62:2840-2847.

49. Landen, C.N., Jr.,et al. 2006. Efficacy and antivascular effects of EphA2 reduction with an agonistic antibody in ovarian cancer. J. Natl. Cancer Inst. 98: $1558-1570$

50. Kiewlich, D., et al. 2006. Anti-EphA2 antibodies decrease EphA2 protein levels in murine CT26 colorectal and human MDA-231 breast tumors but do not inhibit tumor growth. Neoplasia. 8:18-30.

51. Penichet, M.L., et al. 1999. In vivo properties of three human HER2/neu-expressing murine cell lines in immunocompetent mice. Lab. Anim. Sci. 49:179-188.

52. Ursini-Siegel, J., Schade, B., Cardiff, R.D., and Muller, W.J. 2007. Insights from transgenic mouse models of ERBB2-induced breast cancer. Nat. Rev. Cancer. 7:389-397.

53. Tang, C.K., Gong, X.Q., Moscatello, D.K., Wong, A.J., and Lippman, M.E. 2000. Epidermal growth factor receptor vIII enhances tumorigenicity in human breast cancer. Cancer Res. 60:3081-3087.

54. Brandt, R., et al. 2000. Mammary gland specific
hEGF receptor transgene expression induces neoplasia and inhibits differentiation. Oncogene. 19:2129-2137.

55. Larsen, A.B., et al. 2007. Activation of the EGFR gene target EphA2 inhibits epidermal growth factor-induced cancer cell motility. Mol. Cancer Res. 5:283-293.

56. Klijn, J.G., Berns, P.M., Schmitz, P.I., and Foekens, J.A. 1992. The clinical significance of epidermal growth factor receptor (EGF-R) in human breast cancer: a review on 5232 patients. Endocr. Rev. 13:3-17.

57. Klijn, J.G., et al. 1994. The prognostic value of epidermal growth factor receptor (EGF-R) in primary breast cancer: results of a 10 year follow-up study. Breast Cancer Res. Treat. 29:73-83.

58. Wikstrand, C.J., et al. 1995. Monoclonal antibodies against EGFRvIII are tumor specific and react with breast and lung carcinomas and malignant gliomas. Cancer Res. 55:3140-3148.

59. Ge, H., Gong, X., and Tang, C.K. 2002. Evidence of high incidence of EGFRvIII expression and coexpression with EGFR in human invasive breast cancer by laser capture microdissection and immunohistochemical analysis. Int. J. Cancer. 98:357-361.

60. Tsutsui, S., et al. 2003. Prognostic value of the combination of epidermal growth factor receptor and c-erbB-2 in breast cancer. Surgery. 133:219-221.

61. Rae, J.M., et al. 2004. EGFR and EGFRvIII expression in primary breast cancer and cell lines. Breast Cancer Res. Treat. 87:87-95.

62. Cheng, N., and Chen, J. 2001. Tumor necrosis factor-alpha induction of endothelial ephrin A1 expression is mediated by a p38 MAPK- and SAPK/JNKdependent but nuclear factor-kappa B-independent mechanism. J. Biol. Chem. 276:13771-13777.

63. Brantley-Sieders, D.M., et al. 2004. EphA2 receptor tyrosine kinase regulates endothelial cell migration and vascular assembly through phosphoinositide 3-kinase-mediated Rac1 GTPase activation. J. Cell Sci. 117:2037-2049.

64. Bergers, G., et al. 2000. Matrix metalloproteinase-9 triggers the angiogenic switch during carcinogenesis. Nat. Cell Biol. 2:737-744.

65. Brantley, D.M., et al. 2001. Nuclear factor-kappaB (NF-kappaB) regulates proliferation and branching in mouse mammary epithelium. Mol. Biol. Cell. 12:1445-1455.

66. Muraoka-Cook, R.S., et al. 2004. Conditional overexpression of active transforming growth factor beta1 in vivo accelerates metastases of transgenic mammary tumors. Cancer Res 64:9002-9011.

67. Muraoka, R.S., et al. 2002. ErbB2/Neu-induced, cyclin D1-dependent transformation is accelerated in 2 27-haploinsufficient mammary epithelial cells but impaired in p27-null cells. Mol. Cell. Biol. 22:2204-2219.

68. Brantley-Sieders, D.M., Fang, W.B., Hwang, Y., Hicks, D., and Chen, J. 2006. Ephrin-A1 facilitates mammary tumor metastasis through an angiogenesis-dependent mechanism mediated by EphA receptor and vascular endothelial growth factor in mice. Cancer Res. 66:10315-10324.

69. Brummelkamp, T.R., Bernards, R., and Agami, R. 2002. A system for stable expression of short interfering RNAs in mammalian cells. Science. 296:550-553

70. Debnath, J., Muthuswamy, S.K., and Brugge, J.S 2003. Morphogenesis and oncogenesis of MCF$10 \mathrm{~A}$ mammary epithelial acini grown in threedimensional basement membrane cultures. Methods. 30:256-268.

71. Spancake, K.M., et al. 1999. E7-transduced human breast epithelial cells show partial differentiation in three-dimensional culture. Cancer Res. 59:6042-6045. 